\title{
Shock-wave surfing
}

\author{
By S.J. LA URENCE AND R. DEITERDIN G \\ ${ }^{1}$ Institute of Aerodynamics and Flow Technology, Spacecraft Section, German Aerospace \\ Center, Bunsenstraße 10, 37073 Göttingen, Germany \\ ${ }^{2}$ Oak Ridge National Laboratory, P.O. Box 2008 MS6367, Oak Ridge, TN 37831, USA
}

(Received ?? and in revised form ??)

A phenomenon referred to as "shock-wave surfing", in which a body moves in such a way as to follow the shock wave generated by another upstream body, is investigated numerically and theoretically. This process can lead to the downstream body accumulating a significantly higher lateral velocity than would otherwise be possible, and thus is of importance in situations such as meteoroid fragmentation, in which the fragment separation behaviour following disruption is determined to a large extent by aerodynamic effects. The surfing effect is first investigated in the context of interactions between a sphere and a planar oblique shock. Numerical simulations are performed and a simple theoretical model is developed to determine the forces acting on the sphere. A phase-plane description is employed to elucidate features of the system dynamics. The theoretical model is then generalised to the more complex situation of aerodynamic interactions between two spheres, and, through comparisons with further computations, is shown to adequately predict, in particular, the final separation velocity of the surfing sphere in initially touching configurations. Both numerical simulations and theory indicate a strong influence of the body radius ratio on the separation process and predict a critical radius ratio for initially touching fragments that delineates entrainment of the smaller fragment within the larger fragment's shock from expulsion; this critical ratio also results in the most extended surfing. Further, these results show that an earlier prediction for the separation velocity to scale with the square root of the radius ratio does not accurately describe the separation behaviour. The theoretical model is then employed to investigate configurations with varying initial relative sphere positions and velocities. A phase-space description is also shown to be useful in elucidating the dynamics of the sphere-sphere system. With regard to meteoroid fragmentation, it is shown that a large fraction of the variation in the separation behaviour deduced by previous authors from an analysis of terrestrial crater fields can be explained by a combination of surfing and a modest rotation rate of the parent body. Finally, a selection effect for multiple fragments travelling together, e.g., immediately following atmospheric disruption, is predicted, whereby larger fragments repel one another whereas smaller fragments are entrained within the shocks of larger bodies.

\section{Introduction}

The problem of aerodynamic interactions between bodies travelling at hypersonic speeds is an interesting topic of study in its own right, but is also important to our understanding of such phenomena as the fragmentation of meteoritic bodies in the atmospheres of Earth and other planets. The separation behaviour of fragments immediately following atmospheric disruption is determined to a large extent by aerodynamic effects (Passey \& 
Melosh 1980), and this in turn can determine, for instance, the arrangement of craters in the strewn field formed by fragments that survive the atmospheric transit, or the damage produced at the planetary surface by bodies that explode in the atmosphere (Hills \& Goda 1993). For bodies that are disrupted into a limited number of distinct fragments, rather than heavily fragmented into a liquid- or swarm-like mass (Chyba et al. 1993; Svetsov et al. 1995), it is necessary to consider the shock interactions between fragments to gain an accurate picture of the separation process.

The first to consider in detail the separation of distinct meteoroid fragments were Passey \& Melosh (1980), who, by assuming a purely transverse separation due to bow-shock interactions between two fragments of radii, $r_{1} \geqslant r_{2}$, predicted the smaller body to develop a final separation velocity, $V_{T}$, of

$$
V_{T}=\sqrt{C \frac{r_{1}}{r_{2}} \frac{\rho_{a}}{\rho_{m}}} V,
$$

where $\mathrm{V}$ is the velocity of the meteoroid through the atmosphere; $\rho_{a}$ and $\rho_{m}$ are the densities of the atmosphere and the meteoroid, respectively. $C$ is a constant that, through examining various terrestrial crater fields, was determined by these authors to have a value between 0.03 and 2.25. As much of the subsequent work on the topic has involved the modelling of equally sized fragments, (1.1) is often rewritten in the form

$$
V_{T}=\sqrt{C \frac{\rho_{a}}{\rho_{m}}} V .
$$

Here we do so in anticipation that (1.1) does not provide an accurate representation of the scaling of $V_{T}$ with $r_{2} / r_{1}$.

Employing numerical simulations to investigate the symmetrical separation of two identical fragments, Artem'eva \& Shuvalov (1996) determined a value of $C$ of approximately 0.2 ; the results of these authors also showed that the lateral force acting on a trailing body within the shocked region of a leading body is attractive. This confirmed the collimation effect observed experimentally by Schultz \& Sugita (1994), in which individual particles in a debris cloud were seen to "surf" over the cloud shock front and become entrained within the shocked region. Subsequent numerical simulations of up to 27 identical fragments (Artemieva \& Shuvalov 2001) indicated a value of $C$ of approximately unity. Models based on these findings have been used in simulating the breakup of meteoritic bodies in the atmosphere and to draw conclusions, for example, regarding the rate of arrival of small asteroids at the Earth's surface (Bland \& Artemieva 2003, 2006; Artemieva \& Pierazzo 2009). Further work involving the modelling of stationary bodies (Laurence et al. 2007; Barri 2009) has contributed to our understanding of the aerodynamic interactions between fragments of different sizes; however, the effect of relative fragment size on the separation process has still not been properly elucidated. It is one of the intents of this paper to address this deficiency.

The separation model developed by Passey \& Melosh assumes the two fragments, initially lying next to one another, to separate only in the lateral direction. While appropriate for equally sized fragments, for unequally sized bodies possessing differing ballistic coefficients this assumption can no longer be considered valid. The smaller body (which, for brevity, we refer to as the secondary) travels downstream relative to the larger body (the primary) due to its lower ballistic coefficient, as well as laterally due to the repulsive effect of the highly compressed gas lying in the region between the two. A situation can then develop in which the primary bow shock impinges on the secondary; the lateral force remains repulsive, however, as the doubly shocked flow on the body's inner side is at a higher pressure than the singly shocked flow on its outer side. Provided the balance of 

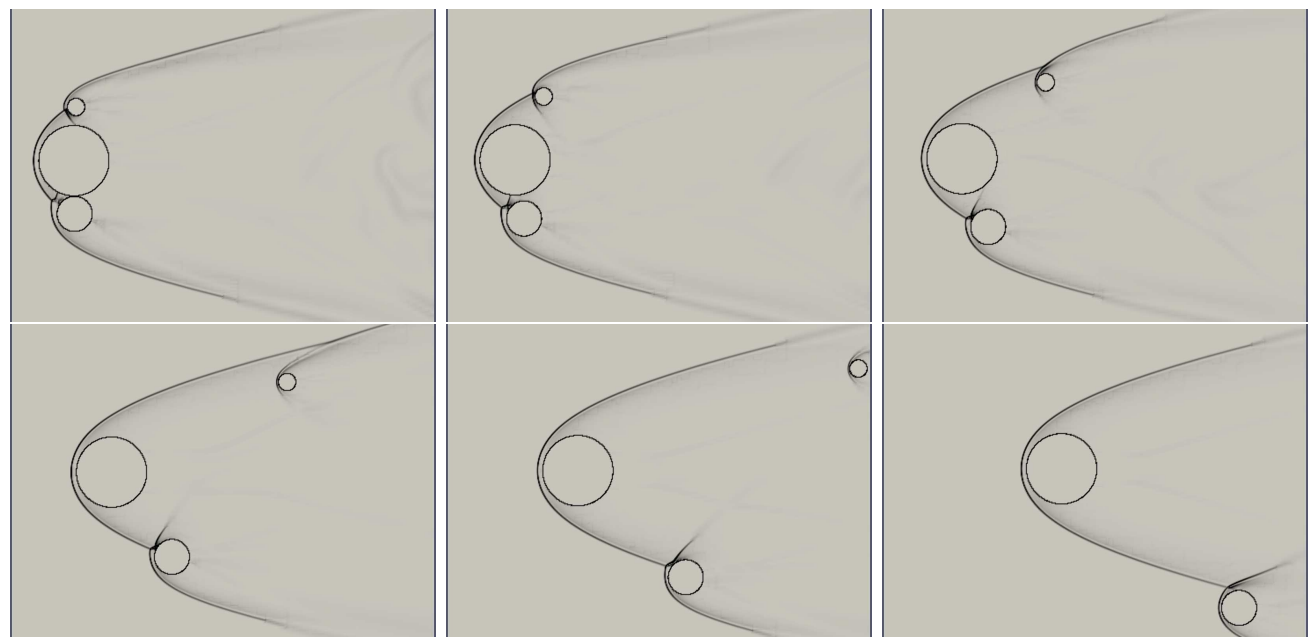

FIGURE 1. "Shock-wave surfing", in which a smaller body rides downstream on the bow-shock created by a larger body. The smaller body can either be entrained in the shock layer (e.g., upper body), or ejected (lower body), as a result.

forces remains correct, the secondary can then follow, or "surf", the primary bow-shock downstream.

An example of such surfing is shown in figure 1, depicting a numerical simulation of three spheres of relative sizes $1,1 / 2$ and $1 / 4$ in a uniform Mach 10 flow. The bowshock from the primary (largest) body initially impinges on each of the two secondaries: the forces on the larger secondary cause it to follow the bow-shock downstream over a considerable distance, gaining significant lateral momentum in the process; the smaller secondary, after surfing a short distance, is entrained within the shock layer. The transverse velocity of the larger secondary when it exits the shock gives a value for $C$ in (1.1) of 0.35 , which compares with a value of 0.04 obtained from a simulation of two equally sized spheres. While these exact values will depend to some extent on the body geometries (note, for example, that Artem'eva \& Shuvalov (1996) obtain $C \approx 0.2$ for two symmetrical hemispheres), the physical phenomena that produce the surfing effect are in no way particular to the spherical configuration considered here. Thus, significantly enhanced separation velocities can also be expected for other geometries. It is also clear that the simple $r_{2} / r_{1}$ scaling indicated by (1.1) cannot be considered to hold over a very large range of radius ratios.

This phenomenon may seem superficially similar to the "surfing" effect referred to earlier, noted by Schultz \& Sugita (1994) of small particles within a debris cloud; but the two are fundamentally different. The particle-surfing in the earlier work consists of movement within the shock layer, and always leads to collimation of the particles within the shocked region. The present phenomenon results from the special conditions created by the interaction of the impinging shock with the secondary body, and may lead to the secondary being either ejected from or entrained within the primary bow-shock.

The simulation shown in figure 1 required approximately $3000 \mathrm{CPU}$ hours to complete on a typical PC-cluster with 12 quad-core nodes. Considering the number of parameters that can be varied in this problem, simulations of this size are not a realistic tool for fully exploring the shock-surfing phenomenon. Thus, to aid in the development of a theoretical model, in $\S 3$ we first consider a simplified version of the surfing problem, namely a sphere interacting with a planar oblique shock. We verify the theoretical model 


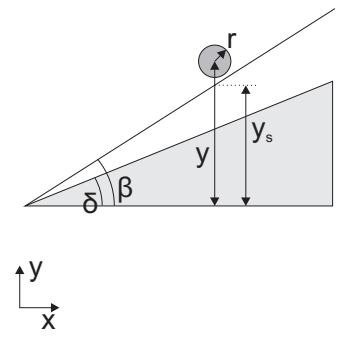

(a)

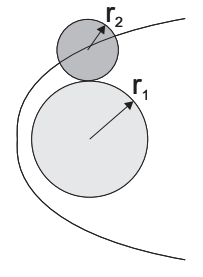

(b)

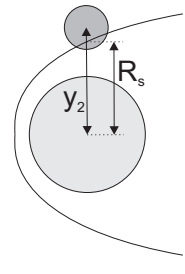

(c)

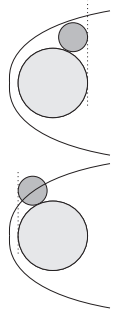

(d)

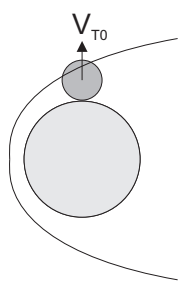

(e)

FIGURE 2. Summary of initial configurations considered in the present work: (a) sphere, initially stationary, interacting with wedge-generated oblique shock (relevant to figures 3-10); (b) spheres initially stationary and touching, with centres axially aligned (figures 11, 12, 15-17, 23); (c) spheres initially stationary, centres aligned axially but with lateral separation (figures 18-21); (d) as in (b) but with rearmost or frontmost points aligned (figure 22-left); (e) as in (b) but with an initial lateral separation velocity (figure 22-right).

with large-scale, high-resolution computations. The developed model is then generalised in $\S 4$ to interactions between two spheres and, after validation of the results with a number of further numerical simulations, used to explore the surfing phenomenon and the separation behaviour in this case. In $\S 5$, a discussion of the significance of these results for the problem of meteoroid fragmentation appears. For the reader's convenience, a schematic summary of the configurations considered in this work is presented in figure 2; for each configuration, the relevant figure numbers are indicated in the caption.

We restrict our attention throughout to spherical geometries in order to avoid the additional complication of induced rotations. The use of such blunt geometries also ensures that viscous contributions to the forces are small, justifying the present inviscid treatment. The gas is treated as perfect throughout: while real-gas effects, including dissociation, vibrational excitation, radiation and ionisation, are important at the flow conditions relevant to meteoroid entry, the influence of these on the pressure, and thus the forces, is generally limited (see, for example, Vincenti \& Kruger (1965) regarding dissociational effects and Artemieva \& Shuvalov (2001) regarding radiative and ablative effects).

\section{Computational modelling}

In this work we are concerned with supersonic flows undergoing full dynamic interaction with rigid solid bodies. Reliable fluid-structure interaction (FSI) simulations in this regime require the coupling of a shock-capturing fluid dynamics solver for the Euler equations with a rigid body solver that considers the Newtonian laws of motion. The construction of numerical methods for shock-driven FSI problems is, in itself, still an area of active research, and the number of available software codes is accordingly small. The discretizations for both the fluid flow and the rigid body dynamics have to be explicit to give time-accurate results, limiting the possible time step size through a CFL stability condition. On the other hand, changes in the fluid domain due to the solid body motion need to be accounted for at every time step. Since several thousand explicit FSI time steps will typically have to be computed in a given simulation, a fast mesh or geometry adjustment is vital.

Our approach to this problem is to employ an embedded boundary method in the fluid solver, in which moving solid structures slide through a fixed Cartesian fluid background 
mesh. The structure is represented implicitly with a scalar level set function that stores in each fluid grid cell the distance to the nearest point on the solid surface. The sign of the distance function then determines which cells are within the valid fluid domain and which are treated as exterior. Exterior cells near the internal boundary are used to prescribe immersed moving wall boundary conditions. The construction of embedded wall boundary conditions, considering the intrinsic velocity of a solid object while avoiding unphysical overshoots in the boundary values, is described in detail by Deiterding (2009). As is characteristic of level set methods (Fedkiw et al. 1999), the Cartesian finite volume stencil is itself not modified, which results in a slight diffusion of the boundary location. We alleviate such errors by dynamic adaptive mesh refinement (AMR) of the Cartesian mesh in the vicinity of the embedded boundary. The refinement approach used is the spatial and temporal multi-level AMR algorithm after Berger \& Colella (1988), designed specifically for the solution of hyperbolic fluid flow problems. The AMR algorithm and the embedded boundary method are implemented in the AMROC (Adaptive Mesh Refinement in Object-oriented $\mathrm{C}++$ ) code that has been parallelised for distributed memory machines (Deiterding 2003). AMROC's framework architecture and its utilisation for fully coupled shock- and detonation-driven FSI problems is described in depth by Deiterding et al. (2005). Further verification and validation results, that demonstrate the ability of the approach to deal with arbitrary structural evolutions while maintaining computational performance and parallel efficiency, are given by Cirak et al. (2007) and Deiterding et al. (2009); validation results for gaseous flows may be found in Deiterding (2005) and Deiterding (2009).

As in our previous work (Laurence et al. 2007), we consider only spherical nonintersecting, rigid bodies, for which the combined signed distance level set function can be easily computed. Following a computational splitting approach, the level set function is re-computed after each time step on the highest level of fluid mesh adaptation, once the equation of motion for each sphere has been integrated with a forward Euler method. The instantaneous forces due to the flow are evaluated by integrating the fluid pressure on each sphere on a high resolution longitude-latitude mesh. In order to calculate numerical fluxes within the finite volume discretization, we utilise the Van Leer flux vector splitting scheme that is extended to second-order accuracy with the MUSCL-Hancock method using a Minmod-limiter in the primitive variables (see Toro (1999) for details). All computations herein were carried out on three-dimensional meshes using a dimensional splitting approach. Since the centres of the spheres are always on the midplane through the domain, visualisations of computational pseudo-schlieren images typically show the symmetry plane normal to the $z$-axis. The computations use one to three additional levels of mesh adaptation with refinement factors of either 2 or 3 , both in space and in time. Local dynamic refinement is applied up to the highest level available along embedded boundaries and shock waves.

\section{Surfing a planar oblique shock}

By considering impingement involving a planar oblique shock rather than a curved primary shock, the complexity of the surfing problem is significantly reduced. The conditions behind the shock are now uniform, so, provided the sphere is sufficiently far from the shock-generating body that reflected shocks have no influence, the only relevant length scale is the sphere radius. Assuming the sphere velocity to be negligible in comparison to that of the free-stream, the force coefficients will then depend only on the free-stream Mach number, the shock angle and the location of the impingement point. At the Mach numbers of interest here, we may also appeal to the Mach number 


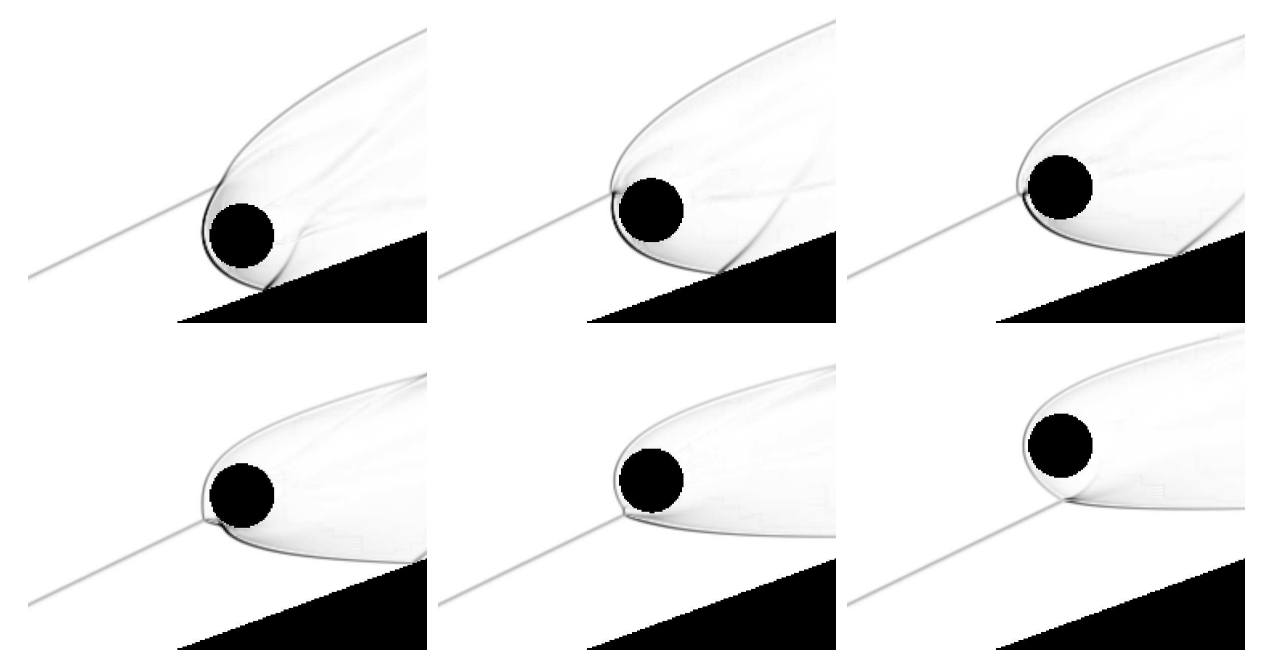

Figure 3. Pseudo-schlieren images of a sphere interacting with the oblique shock produced by a $20^{\circ}$ wedge at Mach 10 .

independence principle (Hayes \& Probstein 1966). Thus, for a given shock angle, we can completely characterise the problem by determining the force coefficients over a range of sphere positions from entirely within to entirely outside the oblique shock. These can then be applied to predict the sphere trajectory for an arbitrary initial position (and velocity).

The definitive work on the interactions between a blunt body and an impinging planar shock was performed by Edney (1968). Depending on the impingement point, a variety of complex flow configurations can be produced. The most important of these is known as the Edney type IV interaction, in which an impinging supersonic jet produces highly elevated local heating and pressure levels. Edney calculated the flowfields individually for several impingement locations, but the complex and varying nature of the shock and shear layer interactions does not lend itself readily to an analysis of the pressure distribution on the body, which is necessary to obtain the desired force coefficients. However, figure 8 of Edney shows that the effect of the impinging structure on the surface pressure distribution is quite localised: away from the impingement point, the pressure soon reverts to roughly the undisturbed profile. Therefore, despite the locally elevated pressure levels, the overall contribution to the integrated forces will be limited. This observation is crucial, as it will allow the development of a simple theoretical model for the forces acting on the sphere, which can then be generalised to the more complex sphere-sphere case. The reduced parameter space in the sphere-wedge configuration, however, makes numerical simulation a feasible tool for characterising the problem, and this is the first focus of this section.

\subsection{Computational evaluation of force coefficients}

In this subsection, we employ the computational model to calculate the drag and lift coefficients of the spherical body interacting with a planar oblique shock in a fully threedimensional flow field. In each simulation, the sphere is initially positioned completely inside the shocked region; once the flow is fully established, it is impulsively started with a lateral velocity of $1 \%$ of the freestream velocity. The aerodynamic forces are calculated by integrating the pressure on the sphere surface at each point along its trajectory. Although the sphere is, in principle, free to respond to the applied forces, the density ratio is set to an artificially high value such that these have a negligible effect on its trajectory. 

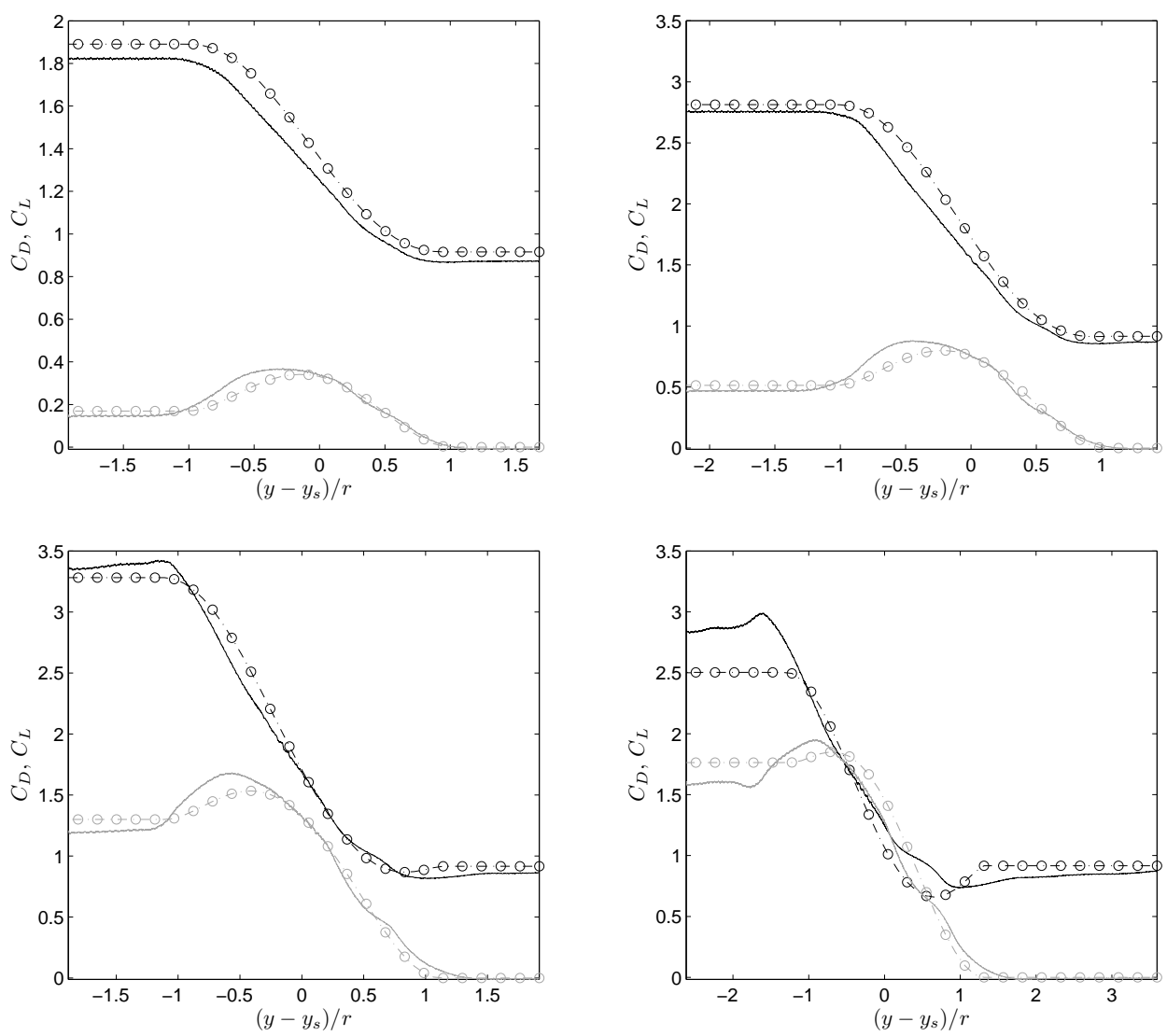

Figure 4. Computational (-) and theoretical $\left(-\cdot--_{-} \cdot-\right.$ ) force coefficients - (dark) drag, (light) lift - for a sphere interacting with a planar oblique shock. The wedge angles are 5, 10, 20, and $30^{\circ}$ (upper left and right, lower left and right, respectively). The abscissa in each case is the normalised lateral displacement from the shock.

The computation is concluded once the sphere has completely exited the influence of the oblique shock.

All computations of the sphere/oblique-shock configuration employ a free-stream Mach number of 10 . The oblique shock is generated by a wedge forming the lower boundary of the computational domain. The extent of the domain depends on the wedge angle: for $\delta=20^{\circ}$, for example, the base grid is $200 \times 140 \times 20$ cells, corresponding to a physical domain of $20 \times 14 \times 2$ sphere diameters. Two levels of additional refinement are applied, each of factor 2; thus, at the highest level the sphere has an effective resolution of 40 cells. A CFL number of either 0.7 or 0.8 is used. All computations were run on 32 quadcore nodes of an IBM BG/P machine; the $\delta=20^{\circ}$ computation, for instance, required $\sim 7,900$ CPU hours and 18,112 time step updates computed on the finest level. In order to quantify the accuracy of the determined force coefficients at this level of resolution, a single refinement study was carried out for a wedge angle of $20^{\circ}$; details of this are provided in $\S 3.4$, together with a visualisation of the refinement strategy for the most refined computation (figure 9 ).

In figure 3 is shown a sequence of computational pseudo-schlieren images from the simulation with a wedge angle of $20^{\circ}$. It is apparent that the mesh is not sufficiently 

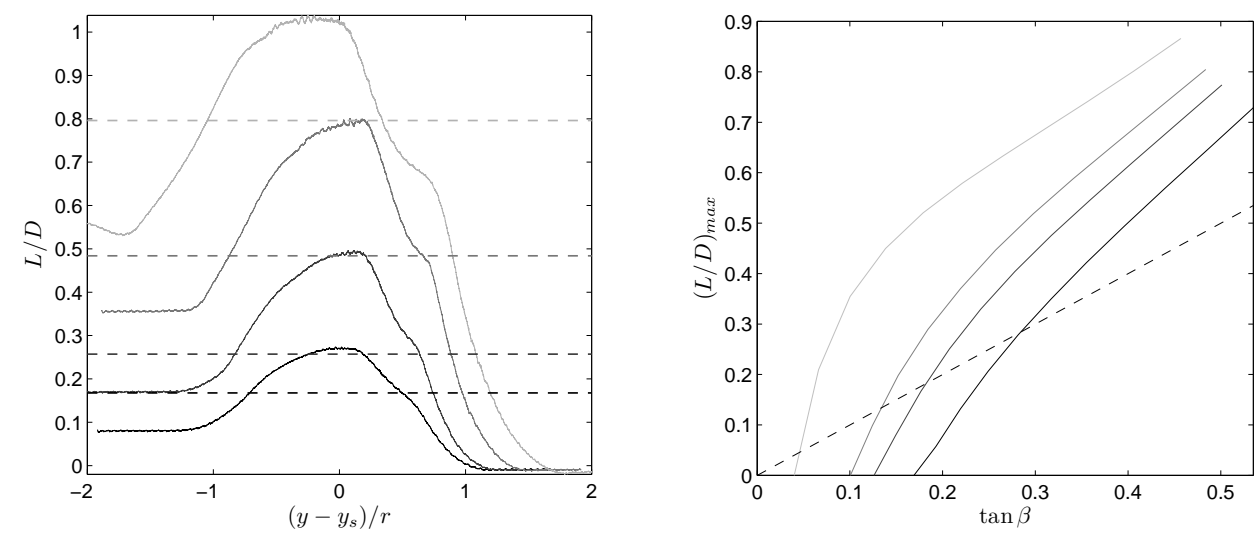

FiguRE 5. (Left) Ratio of lift to drag for sphere/oblique shock interactions, with wedge angles of $5,10,20$, and $30^{\circ}$ (dark to light). The horizontal dashed line for each shade indicates the tangent of the corresponding shock angle. (Right) The maximum $L / D$ value versus the tangent of the shock angle for Mach numbers of (dark through light) $6,8,10$, and 25 . The dashed line corresponds to $(L / D)_{\max }=\tan \beta$.

refined to fully resolve the impinging shock structures in the third and fourth images; however, for the reason outlined earlier, this is unlikely to have an overly significant effect on the force coefficients. The computed drag and lift coefficients from this simulation, together with those for wedge angles of 5,10 and $30^{\circ}$ (corresponding to shock angles of $\beta=25.8,9.5,14.4$, and $38.5^{\circ}$, respectively), are plotted in figure 4 . Also plotted are theoretical profiles, discussed in the following subsection. The abscissa in each case is the lateral displacement (normal to the free-stream flow direction) normalised by the sphere radius, $r$, with the origin being the point at which the plane of the shock passes through the sphere centre. Reading from right to left, corresponding to movement from outside to inside the shock, the drag shows a small dip as the shock first impinges on the rear inner side of the sphere, then rises monotonically, with a small overshoot for larger wedge angles, until the sphere is fully immersed in the shocked region. The lift also rises rapidly once shock impingement begins, but reaches a well-defined maximum before decreasing to its fully immersed value. For the largest wedge angle, the lift is seen to exceed the drag over a small range of positions. Note that, over a broad range of wedge angles $\left(10^{\circ} \lesssim \delta \lesssim 30^{\circ}\right)$, the drag profiles show relatively little variation, with the maximum immersed drag occurring at $\delta \approx 20^{\circ}$; however, the lift profiles vary strongly, with larger wedge angles producing significantly higher maximum lift values.

The lift/drag ratios from these simulations are plotted against the normalised lateral displacement on the left axes of figure 5, together with horizontal lines indicating the tangent of the shock angle in each case. For each wedge angle, this line is seen to intersect the L/D curve in two places. Thus, if the sphere is placed with zero initial velocity at a lateral displacement corresponding to either of these two points, it will remain in the same position relative to the shock, surfing it downstream over the length of the wedge (assuming that the sphere velocity remains negligible compared to the flow velocity). However, only one of these stationary points, that corresponding to a positive $\left(y-y_{s}\right) / r$, is stable. For the other, a small decrease in $y$ will lead to a decreased $\mathrm{L} / \mathrm{D}$, and the sphere will move inside the shock; on the other hand, a small increase in $y$ will lead to an $\mathrm{L} / \mathrm{D}$ greater than $\tan \beta$, and the sphere will be further repulsed. In general, provided the maximum $L / D$ value, $(L / D)_{\max }$, is greater than $\tan \beta$ (this condition is discussed 


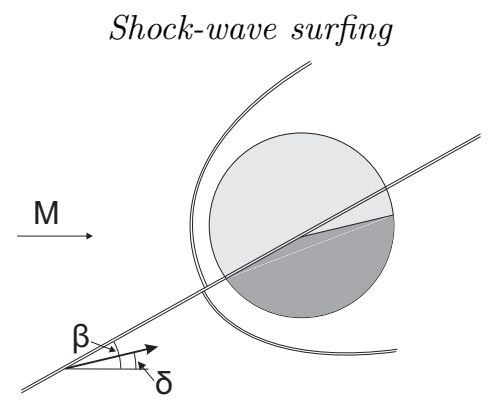

FIgURE 6. Approximation to the impinging flow over the sphere used for the theoretical model: the planar shock is assumed to divide the flow over the sphere into two distinct regions, but producing no interaction with the sphere bow-shock. The flow angle rather than the shock angle is used as the dividing plane on the rear half of the sphere.

shortly), there will always be two such stationary points. This follows from the fact that, outside the shock, $L / D=0$, whilst inside, $L / D=\tan \delta$. As $\tan \delta<\tan \beta$, provided $(L / D)_{\max }>\tan \beta$, the $L / D$ profile must intersect the $\tan \beta$ line (at least) twice.

\subsection{Theoretical modelling of force coefficients}

Given the relatively limited parameter space in the sphere-wedge configuration, numerical simulations are a suitable tool for exploring this problem. It is of interest to see whether a simple theoretical model can yield reasonable results, however, as the development of such a model will be necessary to fully explore the more complex sphere-sphere problem. We begin then, as shown in figure 6, by ignoring the complicated shock and shear-layer interactions near the impingement point, and assume that the impinging shock acts only to divide the flow over the sphere into two separate regions. A rough justification for doing this, appealing to the localised effects of impingement shown in figure 8 of Edney (1968), was given earlier. In each of these regions, we model the surface pressure using the modified Newtonian distribution (Lees 1955):

$$
p= \begin{cases}\left(p_{02}-p_{1}\right) \cos ^{2} \psi+p_{1} & \text { for } \psi \in[0, \pi / 2), \\ p_{1} & \text { for } \psi \in[\pi / 2, \pi),\end{cases}
$$

where outside the impinging shock, $\psi=\theta$ (the zenith angle of the sphere), and inside the shock, $\cos \psi=\cos \theta \cos \delta-\sin \theta \sin \phi \sin \delta$, where $\phi$ and $\delta$ are the azimuthal and flow deflection angles, respectively; $p_{02}$ and $p_{1}$ are the Pitot and static pressures in the relevant region. The drag and lift coefficients are then easily calculated by integrating (3.1) over the surface of the sphere. Note that, as shown in figure 6 , on the rear half of the sphere the flow angle rather than the shock angle is used as the dividing plane between the two flow regions. While there is little difference between results for the two for smaller wedge angles $\left(\delta \leqslant 20^{\circ}\right)$, the use of the shock angle for larger $\delta$ was found to lead to an unrealistically low drag coefficient when the shock was impinging on the inner side of the sphere.

In figure 4 , the force coefficients obtained using this approximate model are compared with computational profiles for several values of the wedge angle. At the largest wedge angle, $\delta=30^{\circ}$, the main features of the profiles are similar, but quantitative agreement is lacking. This is because the modified Newtonian result, while giving a good prediction for the pressure distribution at large Mach numbers, becomes increasingly approximate as the Mach number is decreased. The post-shock Mach number for the $30^{\circ}$ wedge is 2.7 , low enough that errors in the Newtonian result become significant. However, for wedge angles of $20^{\circ}$ (for which the post-shock Mach number is 4.2) or less, agreement is satisfactory. The most notable discrepancy at these smaller wedge angles is an underprediction of 
the lift coefficient between $\left(y-y_{s}\right) / r=-0.5$ and -1 , i.e., where the oblique shock is just impinging upon the outer side of the sphere. From these results, we conclude that the impinging shock interactions have only a limited influence on the drag and lift coefficients, as assumed in the theoretical model.

In the previous subsection, we noted that the sphere can follow the oblique shock downstream provided $(L / D)_{\max } \geqslant \tan \beta$. While we might predict from first principles that the minimum $\beta$ for this condition to hold will increase with decreasing Mach number, the theoretical model provides a convenient method of obtaining an approximate quantitative prediction. On the right axes of figure 5 is plotted $(L / D)_{\max }$ against $\tan \beta$ for four Mach numbers between Mach 6 and 25. The point at which each curve crosses the $(L / D)_{\max }=\tan \beta$ line indicates the minimum $\beta$ for which stable shock-following, i.e., surfing, is possible at that Mach number. We see that for $M=25$, surfing is possible over almost the entire range of $\beta$ down to the Mach angle (at which $(L / D)_{\max }=0$ ). As $M$ is decreased, however, the range of such $\beta$ becomes more limited: at $M=6$, a minimum $\beta$ for stable surfing of $16^{\circ}$ is predicted. The theoretical model cannot be reasonably expected to give accurate results below this Mach number, but we can safely predict that this minimum $\beta$ will continue to increase for lower $M$.

\subsection{Utilisation of force coefficients to predict sphere dynamics}

From the computational drag and lift profiles shown in figure 4, sphere trajectories for various initial positions can be calculated by integrating the set of differential equations

$$
\frac{d \hat{x}}{d \hat{t}}=\hat{v}_{x}, \frac{d \hat{y}}{d \hat{t}}=\hat{v}_{y}, \frac{d \hat{v}_{x}}{d \hat{t}}=\frac{3}{8} C_{D}(\hat{x}, \hat{y}), \frac{d \hat{v}_{y}}{d \hat{t}}=\frac{3}{8} C_{L}(\hat{x}, \hat{y})
$$

where $\hat{x}=x / r$ and $\hat{y}=y / r$ are the nondimensional axial and lateral coordinates, $\hat{t}=\sqrt{\rho_{a} / \rho_{m}} t V / r$ is the nondimensional time; and $\hat{v}_{x}=\sqrt{\rho_{m} / \rho_{a}} v_{x} / V$ and $\hat{v}_{y}=\sqrt{\rho_{m} / \rho_{a}} v_{y} / V$ are the nondimensional axial and lateral sphere velocities, respectively. These trajectories may be considered valid as long as the sphere speed remains negligible compared to the flow speed ahead of the sphere (a criterion that will depend on the density ratio, $\rho_{m} / \rho_{a}$ ). Several trajectories in physical space for a wedge angle of $20^{\circ}$ are plotted in figure 7 ; these are reproduced with the lateral coordinate calculated relative to the shock position, $y_{s}$, in the left plot of figure 8 (the trajectories that penetrate completely into the shocked region are omitted in the latter). For clarity, we have limited ourselves here to $\hat{v}_{x}(0)=\hat{v}_{y}(0)=0$. The stationary points (at which $L / D=\tan \beta$ ) are indicated by dotted lines in both plots, and the shock by double grey lines in figure 7 . The contrasting stabilities of the two stationary points is immediately apparent: a trajectory starting close to the inner point is quickly expelled from the shock, whilst one starting close to the outer point oscillates around it.

The existence of such stationary points and the oscillatory nature of the sphere trajectories suggest that the sphere dynamics could be further elucidated by a phase-plane analysis. Noting then that $C_{D}(\hat{x}, \hat{y})=C_{D}\left(\hat{y}-\hat{y}_{s}\right)=C_{D}(\hat{y}-\tan \beta \hat{x})$, and similarly for $C_{L}$, writing $\eta=\hat{y}-\tan \beta \hat{x}$, the system of equations (3.2) can be reduced to

$$
\frac{d \eta}{d \hat{t}}=v_{\eta}, \frac{d v_{\eta}}{d \hat{t}}=\frac{3}{8}\left[C_{L}(\eta)-\tan \beta C_{D}(\eta)\right]
$$

where $v_{\eta}=\hat{v}_{y}-\tan \beta \hat{v}_{x}$. The phase diagram of (3.3) is shown in the right plot of figure 8 . The stationary points, both lying at $v_{\eta}=0$, are seen to be a centre and a saddle point, respectively; in fact, the phase diagram of (3.3) is very similar to that of a simple pendulum. The bounding neutrally stable orbit indicates the range of initial conditions for which the sphere follows the shock downstream; the sphere trajectories in this region 


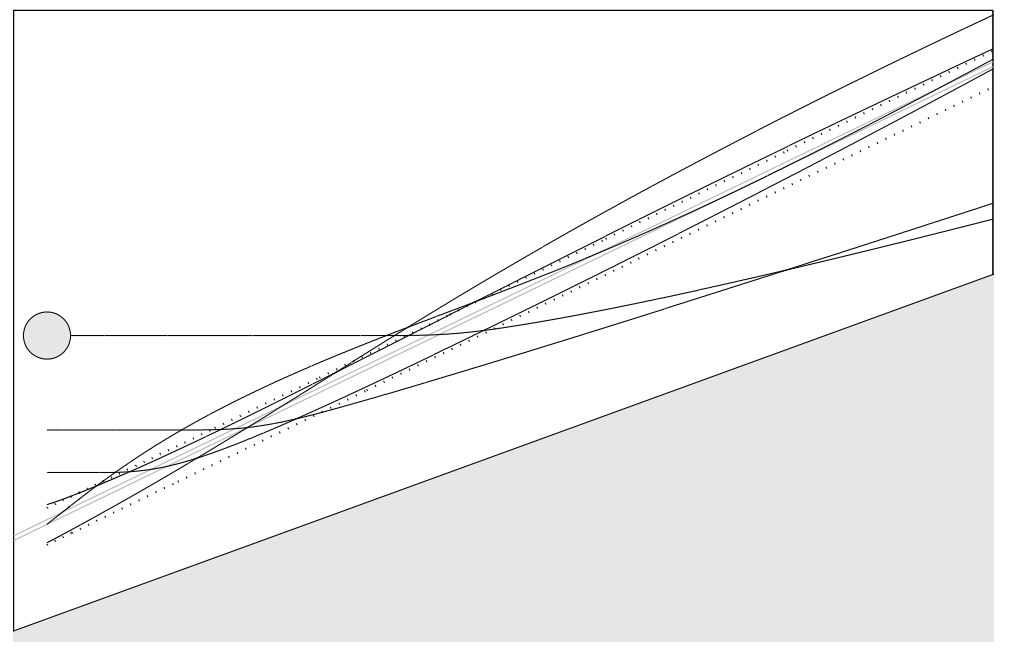

FIgURE 7 . Sphere trajectories for various initial positions and zero initial velocity with $\delta=20^{\circ}$. The shock is indicated by the double grey line; the stationary points (relative to the shock) by dotted lines.
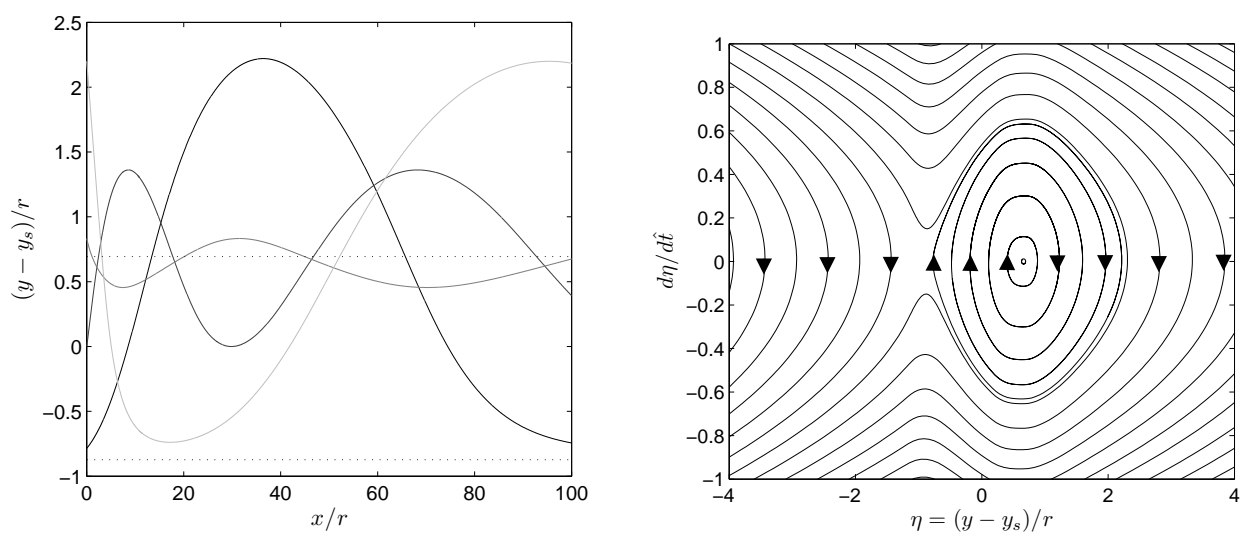

FiguRE 8. Sphere trajectories for $\delta=20^{\circ}$ : (left) in normalised coordinates; (right) in the phase plane. The dotted lines in the left plot indicate the stationary points in the phase diagram.

will oscillate around the stable stationary point with growing wavelengths but no change in amplitude, due to the lack of damping terms in (3.3). For all initial conditions lying outside this orbit, the sphere will penetrate fully into the shocked region and remain there. The range of $\eta$ for shock-following is maximum at $v_{\eta}=0$, extending from the saddle point at $\eta=-0.88$ to $\eta=2.2$. The phase portraits for other wedge angles are qualitatively similar to the one shown: the positions of the stationary points on the $v_{\eta}=0$ axis vary slightly, as may be inferred from figure 5, but all other features are identical.

The nature of the stationary points may also be deduced by considering the eigenvalues 


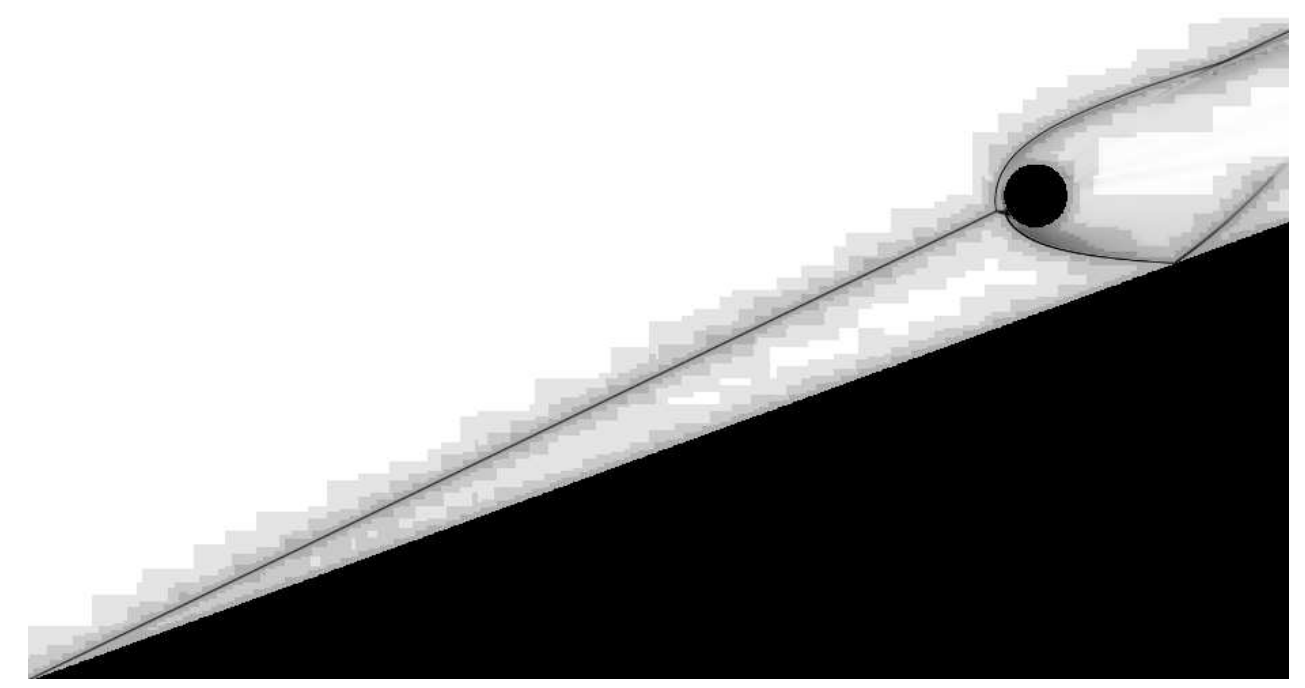

Figure 9. Pseudo-schlieren visualisation of the complete computational domain of the most refined numerical simulation in the refinement study; the successively embedded domains of additional refinement are indicated by the grey levels.

of the Jacobian of (3.3). These are easily shown to be

$$
\lambda= \pm \sqrt{\frac{3}{8}\left(\frac{d C_{L}}{d \eta}-\tan \beta \frac{d C_{D}}{d \eta}\right)} .
$$

Noting from the quotient rule that

$$
\frac{d}{d \eta}\left(\frac{C_{L}}{C_{D}}\right)=\frac{1}{C_{D}^{2}}\left(C_{D} \frac{d C_{L}}{d \eta}-C_{L} \frac{d C_{D}}{d \eta}\right)=\frac{1}{C_{D}}\left(\frac{d C_{L}}{d \eta}-\frac{C_{L}}{C_{D}} \frac{d C_{D}}{d \eta}\right),
$$

and that, at the stationary points, $C_{L} / C_{D}=\tan \beta$, we have

$$
\lambda= \pm \sqrt{\frac{3}{8} C_{D} \frac{d}{d \eta}\left(\frac{C_{L}}{C_{D}}\right)}
$$

where $C_{D}$ and the derivative are evaluated at the relevant point. As $C_{D}>0$, by equating $\eta$ with $\left(y-y_{s}\right) / r$ in figure 5 we see that, at the inner (i.e., $\left.\eta<0\right)$ stationary point, $d\left(C_{L} / C_{D}\right) / d \eta>0$; hence, $\lambda$ are real and of opposite sign, indicating this to be a saddle point. At the outer stationary point, $d\left(C_{L} / C_{D}\right) / d \eta<0$; hence, the eigenvalues are imaginary, indicating that the stationary point in the linearised system is a centre. While it cannot be concluded immediately that the corresponding point in the original system is a centre (a centre in the linearised system may correspond to a stable or unstable node in the nonlinear system), this may be deduced from the absence of damping terms in (3.3).

\subsection{Refinement study}

A refinement study was carried out for a wedge angle of $20^{\circ}$. Up to three additional levels of refinement were applied over the base grid, with all other computational parameters remaining identical. The most refined computation required approximately 50, $250 \mathrm{CPU}$ hours on 32 quad-core nodes of the IBM BG/P, taking 36, 344 time steps updates on the 

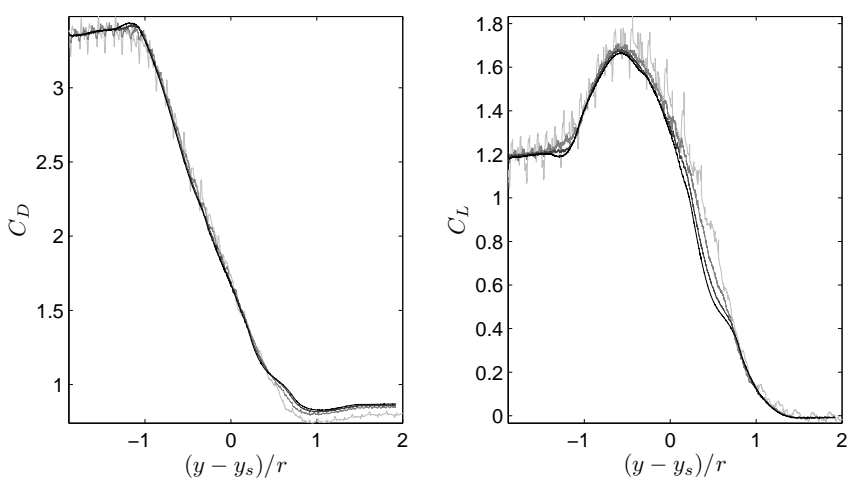

FiguRE 10. Results of refinement study, showing the drag and lift coefficients for zero through three (lightest to darkest) levels of additional refinement over the base grid.

\begin{tabular}{llc} 
& \multicolumn{2}{l}{ RMS change $(\%)$} \\
Additional levels & $C_{D}$ & $C_{L}$ \\
$0-1$ & 2.3 & 6.3 \\
$1-2$ & 1.4 & 3.6 \\
$2-3$ & 0.7 & 2.3
\end{tabular}

TABLE 1. Results of the refinement study, tabulated as the normalised root-mean-square difference between the force coefficients at consecutive levels of refinement (i.e., the standard deviation of the difference normalised by the mean value at the higher level of refinement.)

finest level. Note that on a uniform grid, the computational expense would increase by $2^{4} \equiv 16$ whenever the resolution were doubled. A visualisation of the entire computational domain from this computation, showing both the magnitude of the density gradient and the refinement strategy, is presented in figure 9. Even at this refinement level, the impinging shock structures are not fully resolved; however, increasing the refinement further would be prohibitive in terms of computational cost, and perhaps fruitless insofar as viscous effects would eventually become important at the smallest scales. Regardless, as noted earlier, the effect of such imperfectly resolved shock structures on the force coefficients is unlikely to be problematic.

Results from the refinement study are compared in figure 10: the drag and lift coefficients for each level of refinement are plotted against the normalised lateral displacement of the sphere centre from the shock position. The drag profiles are essentially converged at the highest two levels; there remain small differences in the corresponding lift profiles, but overall convergence may be considered adequate. These results are further summarised in table 1, in which the RMS percentage differences between consecutive levels are tabulated. These differences are seen to progressively decrease in magnitude as the refinement level is increased, though the lift is again slightly more sensitive to changes in refinement at the highest levels. It may be reasonably concluded, however, that the use of two additional refinement levels (as in the computations described earlier) gives satisfactory accuracy in the calculated force coefficients. 

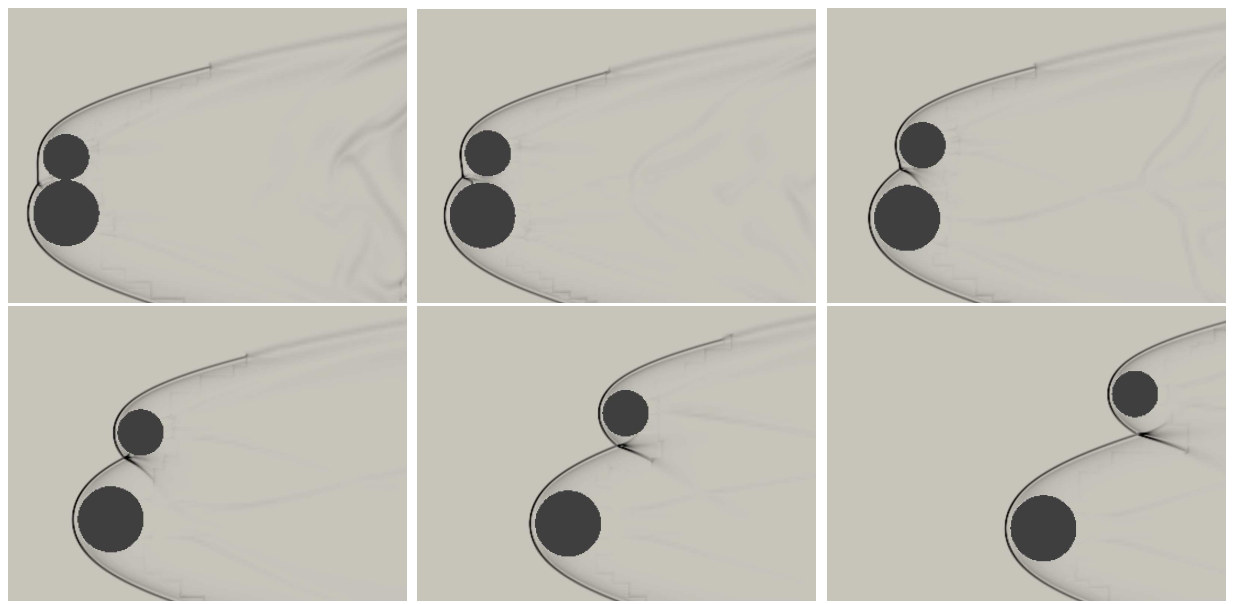

FiguRE 11. Pseudo-schlieren images showing the computed separation behaviour of two initially touching spheres of radius ratio 0.7 at Mach 10 .
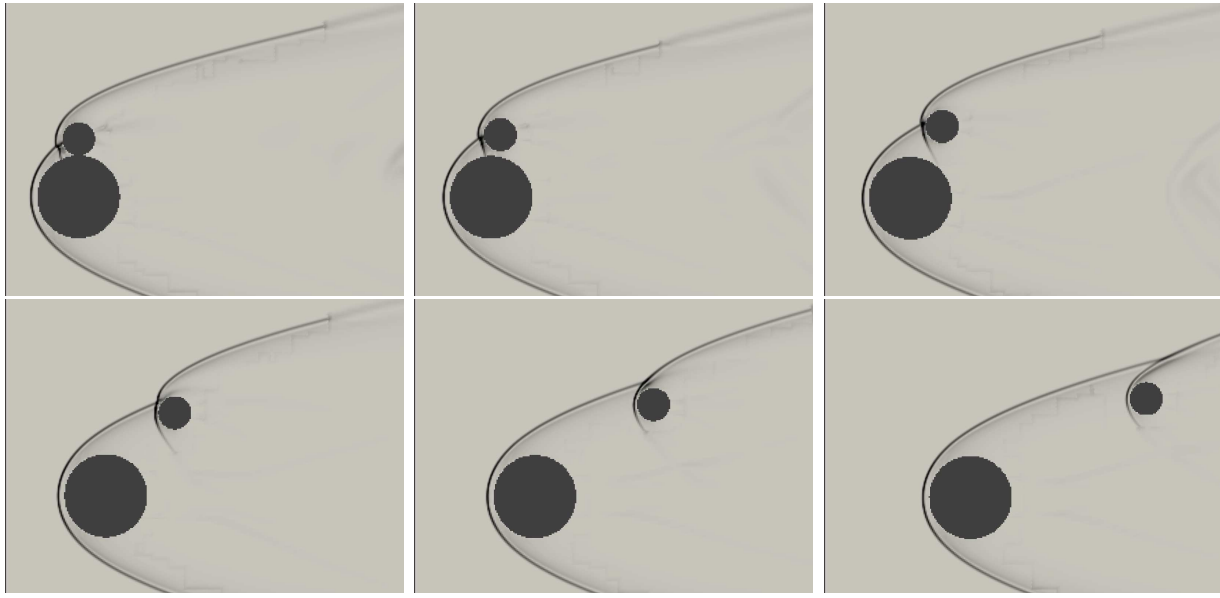

FiguRE 12. Pseudo-schlieren images showing the computed separation of two spheres of radius ratio 0.4 at Mach 10 .

\section{Surfing with spheres}

We now turn to the sphere-sphere problem, more representative of the situation encountered in meteoroid fragmentation. A sense of the important role that the radius ratio plays in determining the separation characteristics may be gained by comparing the visualisation sequences in figures 11 and 12, showing the simulated separations of initially touching sphere pairs with radius ratios of 0.7 and 0.4 , respectively, and that of the $r_{2} / r_{1}=0.5$ case in figure 1 . For $r_{2} / r_{1}=0.7$, surfing takes place over only a short distance and a separation velocity corresponding to $C=0.14$ in (1.1) results (which is modest in comparison to $C=0.35$ calculated for $r_{2} / r_{1}=0.5$, but still significantly higher than $C=0.04$ for $r_{2}=r_{1}$ ). For $r_{2} / r_{1}=0.4$, the secondary is quickly entrained within the shock region, with a maximum lateral velocity corresponding to $C=0.06$. It is apparent that there exists some critical radius ratio in the range $0.4 \leqslant r_{2} / r_{1} \leqslant 0.5$ that delineates entrainment from expulsion for this initially touching configuration. This critical ratio will be the value at which the surfing distance is maximum and the separation velocity close to maximum. 
A number of numerical simulations of the two-sphere configuration were carried out, and are described in $\S 4.3$. However, if, in addition to the radius ratio, both the initial relative positions and velocities of the bodies are allowed to vary, the parameter space of the problem becomes too large to explore computationally; the smallest value of $r_{2} / r_{1}$ and the extent of the physical domain that can be investigated with numerical simulations are also limited. Thus, the following subsection is concerned with developing a theoretical model to determine the impinging force coefficients in the two-sphere surfing problem.

\subsection{Theoretical modelling}

We begin as in the sphere-wedge case, now appealing to the results shown in figure 4, by ignoring the complex flow structures near the impingement point and by assuming that the primary shock acts, as shown in figure 6 , simply to divide the flow over the secondary sphere into two regions (note that the shock angle rather than the flow angle is now used on the rear half of the sphere). The outer region, exposed to the free-stream flow, is modelled as before with the modified Newtonian pressure distribution. Due to the nonuniformity of the flow conditions behind the bow-shock, however, dealing with the inner region is now more difficult. Assuming the impinging shock angle is known, the flow conditions immediately behind the shock (in particular the Pitot pressure and the flow angle) are also known, but approximations must be made further inside the shocked region.

In Laurence et al. (2007), it was shown that the hypersonic blast-wave analogy, when combined with the modified Newtonian theory, gives a good approximation to the forces, and by extension the pressure distribution, acting on a spherical secondary inside a primary bow-shock. Thus, here we use a similar idea for the inner side of the secondary: the pressure at any point on the surface is assumed to follow the modified Newtonian result, (3.1), with the Pitot pressure and flow direction corresponding to the local conditions in the blast-wave solution. The blast-wave results are modified slightly, however, in the form of a scaling that takes into account the known conditions immediately behind the shock (through the oblique shock relations). The static pressure, for example, is given by $p=p_{b w}(x, y) p_{o s}(M, \beta) / p_{b w}\left(x, R_{s}\right)$, where $p_{b w}$ and $p_{o s}$ are the blast-wave and oblique shock pressures, and $R_{s}(x)$ is the shock radius, which, along with the shock angle $\beta(x)$, is assumed to be known; $x$ and $y$ are the axial and lateral coordinates, respectively. The use of the oblique shock relations is intended to address the limited range of validity of the blast-wave solution at finite Mach numbers, in particular at distances downstream for which the strong shock assumption, $M \sin \beta \gg 1$, can no longer be considered valid (see, for example, Laurence (2006)).

With the pressure distribution thus defined, the drag and lift coefficients can be calculated by integrating this over the surface of the secondary, as before. To simplify the integration, the following approximation to the blast-wave solution is made. While an analytical solution to this problem is available (Sedov 1959), it is not an explicit relation for the flow variables. In particular, the relation between the nondimensional lateral flow velocity, $\hat{u}=(\gamma+1) u /(y V / x)$, and the similarity variable, $\eta \propto y / \sqrt{x}$, is

$$
\left(\frac{\eta_{0}}{\eta}\right)^{2}=\hat{u}(\gamma+1-\gamma \hat{u})\left(\frac{2 \gamma \hat{u}-\gamma-1}{\gamma-1}\right)^{-(\gamma-1) / \gamma}
$$

where $\eta / \eta_{0}=y / R_{s}$. The nondimensional density and pressure are both functions of $\hat{u}$ and $\gamma$ alone. However, the product $\hat{u}(\gamma+1-\gamma \hat{u})$ in (4.1) is very close to unity; thus, a good 


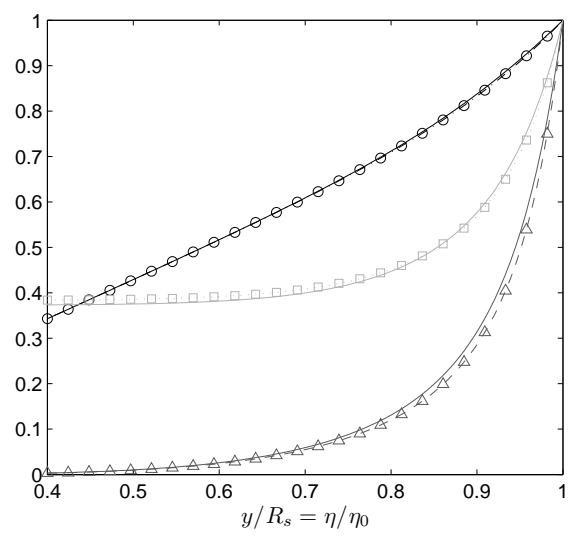

FIGURE 13. Similarity solution to the axisymmetric blast wave problem: (solid) exact solution; (symbols) approximation using (4.2). The flow variables are: $\circ, u / u_{s} ; \triangle, \rho / \rho_{s} ; \square, p / p_{s}$, where the subscript $s$ refers to conditions immediately behind the shock.

approximation to $\hat{u}$ is given by

$$
\hat{u}=\frac{1}{2 \gamma}\left[(\gamma-1)\left(\eta / \eta_{0}\right)^{2 \gamma /(\gamma-1)}+\gamma+1\right] .
$$

Plots of the approximate and exact solutions are shown in 13; considering the other simplifications made in the model, agreement is certainly close enough to justify the use of this approximation.

\subsection{Numerical simulations}

To gauge the performance of the theoretical model, a set of numerical simulations of the two-sphere problem was performed. The simulations are of two types. In the first, the computational methodology is similar to that described in $\S 3$, but with a primary sphere replacing the wedge. The secondary sphere is initially positioned completely inside the primary shock, and, once the flow is established, is impulsively started with a lateral velocity of $1 \%$ of the free-stream velocity. The forces are calculated numerically at each point along the trajectory until the sphere is completely outside the shock. In the second group of simulations, examples of which are shown in figures 11 and 12, the spheres are initially touching one another and, once the flow is established, are released at the same instant with zero initial velocities and subsequently allowed to move freely according to the forces experienced. These latter computations are intended to test the ability of the theoretical model to simulate the fragment separation problem, whereas the first group was performed to give a more complete picture of the performance of the theoretical model in predicting the impinging secondary forces.

Details of the computations are as follows. For the impulsively started simulations, the size of the computational domain depends on the downstream displacement of the secondary. The maximum displacement considered is 4 primary diameters (centre-to-centre); for this simulation, the base grid is $130 \times 100 \times 50$, corresponding to a physical domain of $6.5 \times 5.0 \times 2.5$ primary diameters. For the majority of the simulations, $r_{2} / r_{1}=0.5$; for these, two additional levels of refinement of factor 2 are used, resulting in an effective resolution for the primary of 80 cells at the highest level. For the single $r_{2} / r_{1}=0.25$ simulation, a refinement factor of 3 at the highest level is employed. For all impulsively started simulations, the Mach number is 25 and the CFL number 0.8. The computational overhead depends on the particular geometry; for the simulation with the largest physical do- 
main referred to earlier, 26,200 CPU hours on 256 cores of an IBM BG/P machine were required to perform 34,396 time steps on the finest level.

For the free-flying simulations, a base grid of $130 \times 105 \times 50$, corresponding to a physical domain of $6.5 \times 5.25 \times 2.5$ primary diameters, is employed with a single level of refinement of factor 3 . At the refined level, the effective diameter of the primary sphere is 60 cells. The density ratio, $\rho_{m} / \rho_{a}$, is 1000 , which is a compromise between computational time and a desire that the maximum sphere velocities remain sufficiently low that the results can be considered independent of $\rho_{m} / \rho_{a}$. This choice of value means that the maximum velocity of the secondary is typically limited to $10 \%$ of the free-stream velocity during the time period of interest. A CFL number of 0.8 is used throughout; each computation required only $\sim 100 \mathrm{CPU}$ hours on two cores of a quad-core $2.8 \mathrm{GHz}$ Linux workstation. The single simulation shown in figure 1 is slightly more refined, with the effective resolution of the primary being 80 cells. Two Mach numbers of 10 and 25 are considered and, for each, the radius ratio is varied over a range of values between 0.3 and 1 . For all free-flying computations, the spheres are initially positioned with their centres at the same axial location and with their lateral edges just touching (figure $2 b$ ).

\subsection{Comparison of theoretical and computational results}

In this subsection we assess the performance of the theoretical model by comparing its predictions with the results of the numerical simulations just described. To provide an appropriate comparison, the primary shock profile in the theoretical calculations was specified using a polynomial curve-fit to the computational shock. The surface dividing the flow regions on the secondary in the theoretical model was calculated according to the shock angle at the point of intersection with the sphere; the shock was then assumed to be planar in the axial direction, but shock curvature in the transverse direction was accounted for. In the free-flying simulations, since the theoretical model does not provide any means of predicting the motion of the primary body, this was taken in each case from the corresponding computation (except for the $r_{2}=r_{1}$ case, for which symmetry could be used.) Less ad-hoc methods for dealing with the primary shock shape and the primary motion are discussed later in this article.

In figure 14 are presented computational and theoretical results from the impulsively started simulations. The drag and lift coefficients are plotted against the normalised lateral displacement from the shock (with $R_{s}$ being the effective shock radius at the axial displacement corresponding to the secondary centre). The computational profiles for $y-R_{s}>0$ are seen to be similar to the corresponding sphere-wedge curves shown in figure 4 . As the secondary moves further inside the shock, however, the sharply decreasing flow density (see figure 13) means that the forces quickly drop away from their peak values, with the drag coefficient now also achieving a well-defined maximum. An extensive analysis of the nature of the forces inside the shock may be found in Laurence et al. (2007). Agreement between theoretical and computational results is satisfactory, especially considering the number of simplifying assumptions in the theoretical model. The most noticeable discrepancy is the theoretical prediction for the forces to drop more rapidly for $y-R_{s}<0$. A similar effect was observed in Laurence et al., and was attributed to the strong entropy wake in the blast-wave approximation.

Turning now to the the free-flying simulations, theoretical and computational trajectories for two cases, $r_{2} / r_{1}=0.5$ and 0.6 , both at $M=25$, are shown in figure 15 , and the $x$ and $y$ displacements as functions of time are plotted for the smaller radius ratio in figure 16. From these displacement profiles, it is clear that the theoretical model underpredicts both the drag and the lift in the early stages of the motion, when there is only a small separation between the spheres. This was also clear from a comparison of the 

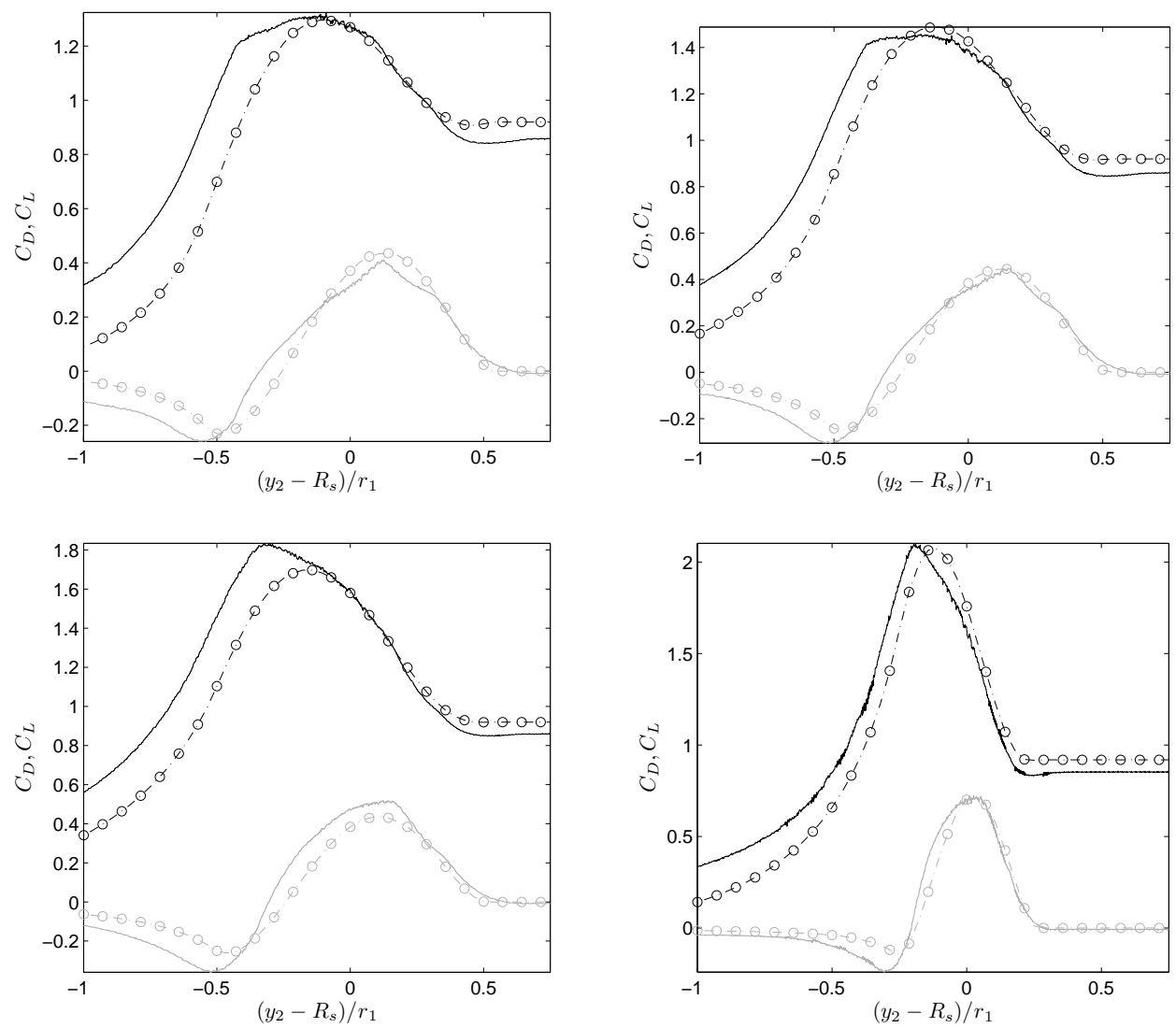

FiguRE 14. Impinging drag (dark) and lift (light) coefficients as functions of the normalised lateral displacement for impulsively started two-sphere simulations at $M=25$ : (upper left) $r_{2} / r_{1}=0.5, \hat{x}=\left(x_{2}-x_{1}\right) / r_{1}=1.5$; (upper right) $r_{2} / r_{1}=0.5, \hat{x}=3.0$; (lower left) $r_{2} / r_{1}=0.5$, $\hat{x}=8.0 ;$ (lower right) $r_{2} / r_{1}=0.25, \hat{x}=3.0 ;-$, computation; - - - - - - - , theory.

theoretical and numerical forces, and is not surprising: not only is the initial shock angle sufficiently large $\left(\sim 40^{\circ}\right)$ that the results of the theoretical model are questionable even for the simpler sphere-wedge configuration (see figure 4), but, in addition, in the physical flow field there is a region of highly compressed gas between the two bodies that augments both the lift and the drag; this feature is not present in the theoretical model. Despite this initial discrepancy, however, the computational and theoretical trajectories lie close to one another, indicating that at least the ratio of the lift to the drag is well captured. Once some separation between the bodies has developed, the theoretical model performs as indicated in figure 14 .

Final computed separation velocities for the complete set of free-flying simulations are compared to the corresponding theoretical values in figure 17. The normalised velocity $V_{T}^{\prime}=\sqrt{\rho_{m} / \rho_{a}} V_{T} / V$, equal to $\sqrt{C}$ in (1.2), is plotted separately against the radius ratio for each of the Mach numbers investigated. As $r_{2} / r_{1}$ is decreased from 1 , the computational profiles are seen to rise increasingly steeply until the critical radius ratio of approximately 0.45 is reached, at which point $V_{T}^{\prime}$ drops sharply. This drop indicates a transition from expulsion of the secondary to entrainment within the primary shock (note that, as the separation velocity is not well-defined for entrainment, the plotted value of $V_{T}^{\prime}$ in this 

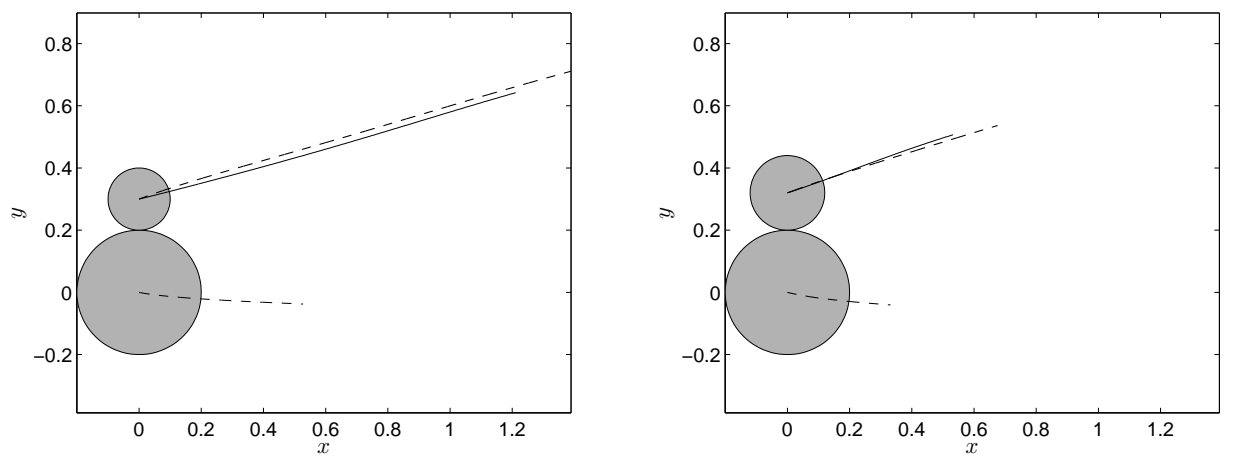

FiguRE 15. Computational (dashed) and theoretical (solid) separation trajectories for radius ratios of $r_{2} / r_{1}=0.5$ (left) and 0.6 (right), at a Mach number of $M=25$. Both trajectories are calculated until the time at which the theoretical secondary leaves the primary shock.
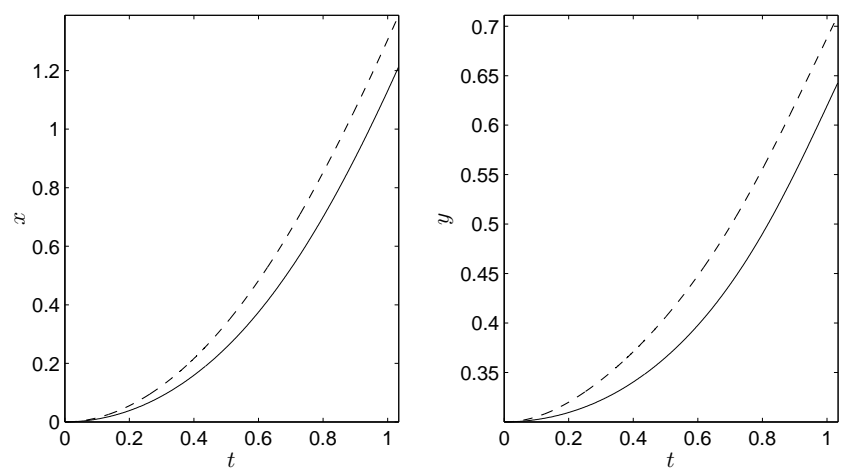

Figure 16. Computational (dashed) and theoretical (solid) secondary displacements for $r_{2} / r_{1}=0.5$ and $M=25$ (corresponding to the left trajectory in figure 15.)

case corresponds to the maximum lateral velocity reached by the secondary.) Although it is not clear from figure 17, a marginally smaller critical $r_{2} / r_{1}$ occurs for $M=25$, due in part to the slightly smaller primary shock radius and in part to the stronger bow-shock producing a larger secondary lift coefficient at the higher Mach number.

The theoretical separation velocities show good agreement with computations at $r_{2} / r_{1}=1$ (which must be considered somewhat fortuitous), but then rise more gradually as the radius ratio is decreased. This is consistent with the deficit in the theoretical lift observed in the initial stages of the separation, as exemplified in figure 16. Slightly higher critical radius ratios, of 0.52 and 0.48 , respectively, for $M=10$ and 25 , are predicted by the model than are observed in computations. Also, as $r_{2} / r_{1}$ is decreased below this critical value, the theoretical model predicts the maximum lateral velocity to drop more rapidly. Agreement for the higher Mach number of the two is superior; this is to be expected, as the blast wave approximation assumes $M \rightarrow \infty$. According to (1.1), the plotted normalised velocity should scale as $\sqrt{r_{1} / r_{2}}$, and such curves are also shown in figure 17 . This scaling is seen to give a poor approximation to the separation behaviour, however, and clearly does not predict a critical radius ratio that separates entrainment from expulsion.

We conclude from these comparisons that the theoretical model developed here, while limited in its ability to predict the forces accurately when the separation between the two 

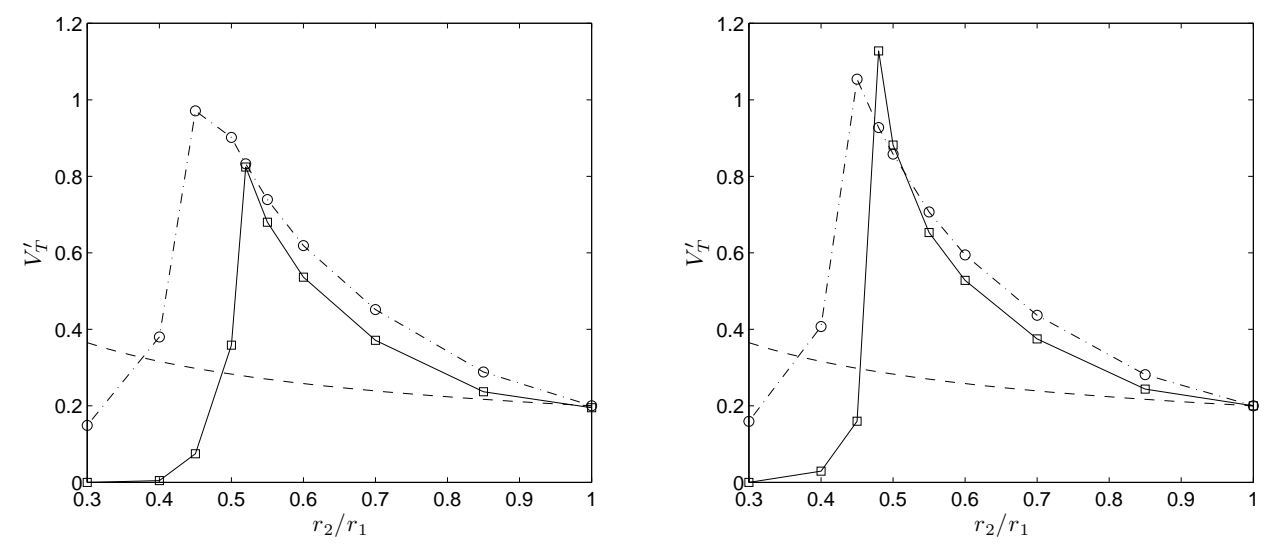

FiguRE 17. Normalised separation velocities, $V_{T}^{\prime}=\sqrt{\rho_{m} / \rho_{a}} V_{T} / V$, for two initially touching spheres with centres axially aligned, for Mach numbers of 10 (left) and 25 (right): - $\square-$, theory; $-\cdot--_{--\cdot}$, numerical simulations; --- , scaling indicated by (1.1). Note that for $M=10$, $r_{2} / r_{1}=0.45$, the primary shock was still impinging upon the secondary when it left the computational domain.

bodies is small, provides satisfactory predictive ability elsewhere. Furthermore, it gives an adequate approximation to the secondary trajectory and to the final separation velocity in simulating the separation of spherical fragments, especially at the Mach numbers relevant to meteoroid fragmentation (i.e., $M=25$ and higher).

In the following two subsections then, the theoretical model is employed to further investigate the effects of varying the radius ratio, the initial positions, and the relative initial velocities of the spheres. To avoid reliance on the results of numerical simulations, the correlation of Billig (1967) is now used in place of a computational profile for the primary bow-shock shape. Also, the primary sphere motion is assumed to be unaffected by the presence of the secondary, i.e., constant values of the primary drag and lift coefficients of $C_{D 1}=0.9$ and $C_{L 1}=0$ are assigned. For initially separated spheres, this is a reasonable assumption. For initially touching spheres, the influence of the secondary on the primary drag in the free-flying computations was found to be generally small ( $\lesssim 13 \%$ for $r_{2} / r_{1} \lesssim$ $0.7)$; thus, the assumption of zero lift here is approximately equivalent to modelling a symmetric three-body configuration with two secondaries. The differences between this and the original two-body configuration are described shortly. All calculations described in these subsections, unless stated otherwise, employ a Mach number of 25.

\subsection{System dynamics in sphere-sphere surfing}

We now investigate the effect of varying the initial lateral separation of the spheres (figure 2c), in particular to determine whether a range of stable surfing behaviour, as was found for the sphere-wedge arrangement, can be established. For the initially touching two-sphere configurations considered thus far, surfing was found in general to be unstable. This is because for a secondary sufficiently large to avoid entrainment $\left(r_{2} / r_{1} \gtrsim 0.45\right)$, the lateral momentum developed during the impingement phase is large enough that, as the secondary exits the shock, the flow cannot bend its trajectory around more quickly than the shock angle is decreasing. By varying the initial lateral separation, however, the initial repulsion for large secondaries can be decreased; also, surfing becomes possible for smaller radius ratios (note that the sphere-sphere behaviour in the limit $r_{2} / r_{1} \rightarrow 0$, and for small axial velocities, will tend locally to that of the sphere-wedge arrangement). 

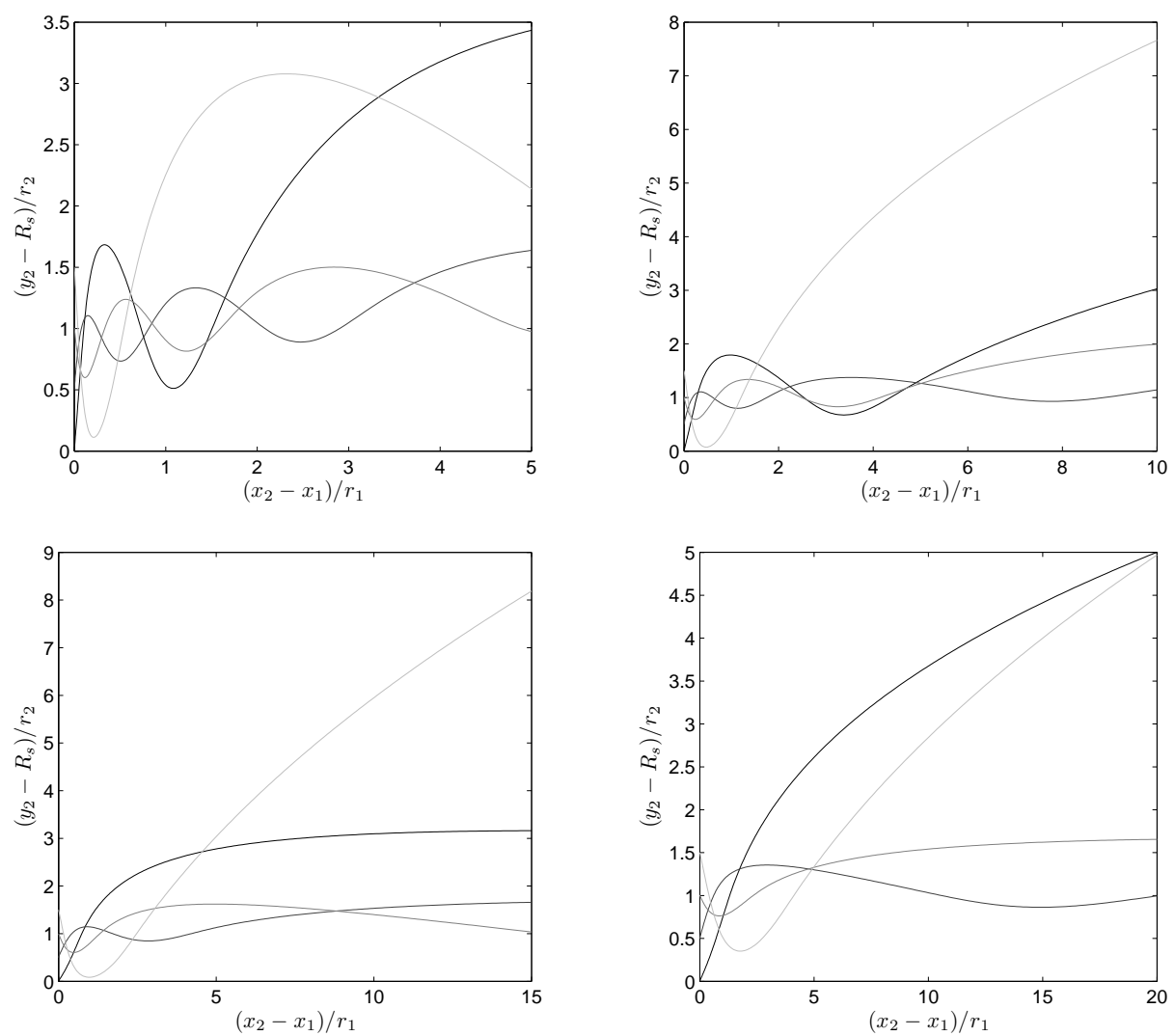

FIGURE 18. Theoretical secondary trajectories in normalised coordinates for various initial lateral positions, for radius ratios of $r_{2} / r_{1}=0.05$ (upper left), 0.1 (upper right), 0.2 (lower left), and 0.5 (lower right), all at Mach 25. Note the differing horizontal and vertical scales.

Thus, it should be possible to reproduce surfing behaviour similar to that observed in the earlier configuration.

\subsubsection{Sphere trajectories}

In figure 18 are plotted secondary trajectories for four different radius ratios: $r_{2} / r_{1}=0.05$, $0.1,0.2$, and 0.5 , each with four choices of the initial lateral position (relative to the shock). These are obtained by integrating (3.2) for each of the two spheres. For the primary, having already assumed the force coefficients to be constant, the integration is trivial. For the secondary, now $\hat{x}=\left(x_{2}-x_{1}\right) / r_{1}$ and $\hat{y}=\left(y_{2}-y_{1}\right) / r_{1}$ in the evaluation of $C_{D}$ and $C_{L}$. In all the cases shown, the secondary and primary centres are initially aligned in the axial direction and the initial velocities are zero. The abscissa in each plot is the normalised axial distance between sphere centres, $\hat{x}$; the ordinate is the lateral distance between the secondary centre and the shock radius, $R_{s}$, normalised by the secondary radius. Thus, the secondary is completely outside the shock for $\left(y_{2}-R_{s}\right) / r_{2}>1 / \cos \beta$. This scaling was chosen as it is the most convenient for the following analysis.

For all $r_{2} / r_{1}$, the minimum initial separation considered of $y_{20}-R_{s}=0$ (at which the initial lateral force is close to a maximum) results in the secondary being quickly ejected from the shock. For the two smaller $r_{2} / r_{1}$, the aerodynamic forces acting in the undisturbed free-stream are sufficient to turn the trajectory so that the secondary 
re-enters the shocked region, only for it to be ejected once again (for $r_{2} / r_{1}=0.05$, a further re-entry occurs at $\hat{x} \approx 20$ ). For the two larger radius ratios, the secondary remains outside the shock after the first ejection. For the maximum initial separation shown, $\left(y_{20}-R_{s}\right) / r_{2}=1.5$, the secondaries begin their trajectories outside the primary shock. The axial momentum developed in the free-stream allows the body to penetrate deeply into the shock in each case, but the large repulsive forces then encountered cause it to be strongly expelled. Increasing the initial separation further to $\left(y_{20}-R_{s}\right) / r_{2}=2$ results in the secondary penetrating completely inside the shock and becoming entrained for all but the largest radius ratio. For the two intermediate initial separations, $\left(y_{20}-R_{s}\right) / r_{2}=0.5$ and 1.0, the secondary motion is similar to the oscillatory behaviour observed for spherewedge interactions, with the sphere dipping in and out of the shocked region, but typically failing to develop sufficient lateral momentum to be decisively ejected. The oscillation wavelength increases with $r_{2} / r_{1}$, and, because of the decreasing shock angle with $\hat{x}$, does so more rapidly than according to the linear relationship that is exhibited for a planar shock.

\subsubsection{Governing equations in phase space}

It is clear from the trajectories just discussed that the sphere-sphere system behaviour is at least to some extent similar to that of the sphere-wedge configuration. Unfortunately, now that the shock angle $\beta$ is a function of $\hat{x}$, the flow similarity that enabled (3.2) to be reduced to (3.3) no longer exists, complicating efforts to derive a comprehensive phasespace description. We proceed then as follows.

We begin by assuming that the Mach number and radius ratio are given, and that the spheres are initially stationary. By analogy with the reasoning that led to (3.3), we now write $\eta=\left(y_{2}-R_{s}\right) / r_{2}$, so that $v_{\eta}=d \eta / d \hat{t}=\hat{v}_{y 2}-\hat{v}_{y 1}-\tan \beta\left(\hat{v}_{x 2}-\hat{v}_{x 1}\right)$, where $\hat{t}=\sqrt{\rho_{a} / \rho_{m}} t V / r_{2}$. The analogous system of equations to (3.3) is then

$$
\begin{aligned}
\frac{d \eta}{d \hat{t}} & =v_{\eta}, \\
\frac{d v_{\eta}}{d \hat{t}} & =\underbrace{\frac{3}{8}\left[C_{L 2}(\beta, \eta)-\frac{r_{2}}{r_{1}} C_{L 1}-\tan \beta\left(C_{D 2}(\beta, \eta)-\frac{r_{2}}{r_{1}} C_{D 1}\right)\right]}-\underbrace{\frac{\hat{v}_{x 2}-\hat{v}_{x 1}}{\cos ^{2} \beta} \frac{d \beta}{d \hat{t}}}, \\
\frac{d \beta}{d \hat{t}} & =\frac{r_{2}}{r_{1}}\left(\hat{v}_{x 2}-\hat{v}_{x 1}\right) \frac{d \beta}{d \hat{x}},
\end{aligned}
$$

where any newly introduced notation has its obvious meaning. As discussed earlier, $C_{D 1}$ and $C_{L 1}$ are assumed constant; in particular $C_{L 1}=0$. Since $\beta$ is a single-valued function of $\hat{x}$, we can easily reverse this dependency and write $\hat{x}=\hat{x}(\beta)$; in this case, the derivative $d \beta / d \hat{x}$ in $(4.3 c)$ becomes $(d \hat{x} / d \beta)^{-1}$. However (4.3) is prevented from being an autonomous system for $\eta, v_{\eta}$, and $\beta$ by the appearance of $\hat{v}_{x 2}-\hat{v}_{x 1}$ in $(4.3 b)$ and $(4.3 c)$. In general, this expression, representing an integrated drag coefficient, depends on $\hat{t}, \eta(0)$, and $\beta(0)$. Nor is it reasonable to simply ignore this problem by neglecting the relevant term in (4.3b). To show this, in figure 19 are plotted the two underbracketed terms in $(4.3 b)$ (which we refer to as the force-coefficient and shock-curvature terms, respectively) versus $\hat{x}$ for the $r_{2} / r_{1}=0.05$ and 0.5 trajectories from figure 18 . Except for the initial stages of the motion, the magnitudes of the two terms are seen to be comparable. However, if we limit ourselves to a single initial axial position, characterised by $\beta(0)$, the value of $\hat{v}_{x 2}-\hat{v}_{x 1}$ should vary but weakly with the initial lateral position, $\eta(0)$, and may then be approximated as a function only of $\beta$ (or of $\hat{t}$ - the two are now equivalent). If this approximation is reasonable, the shock-curvature term profiles in figure 19 should be roughly independent of $y_{20}$. For $r_{2} / r_{1}=0.05$, this is indeed true. For $r_{2} / r_{1}=0.5$, the 

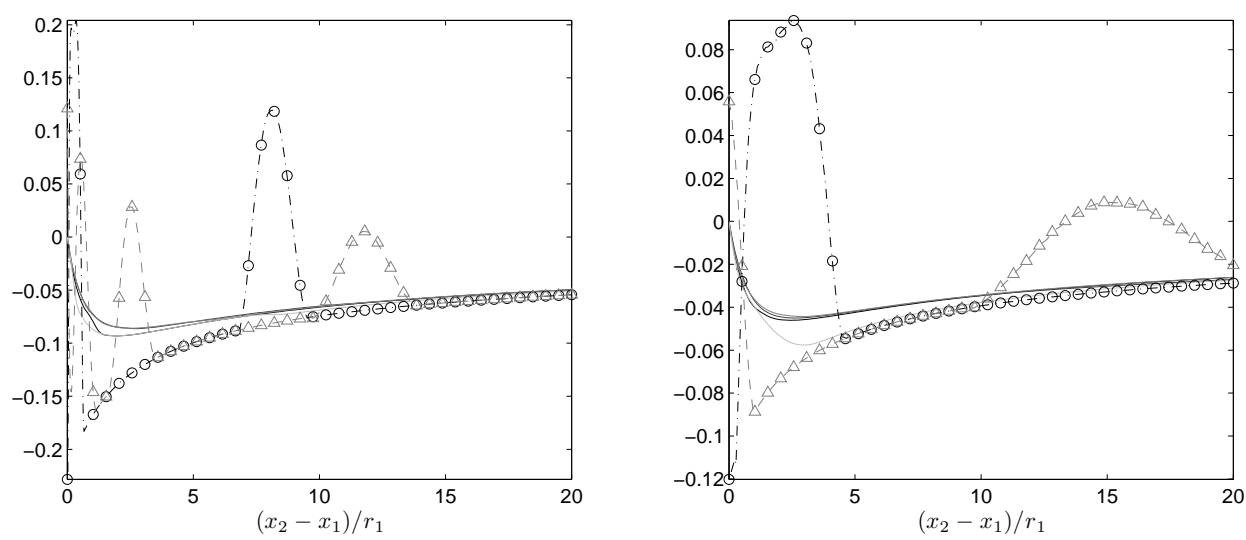

FiguRE 19. Terms in equation (4.3b) for the $r_{2} / r_{1}=0.05$ (left) and 0.5 (right) trajectories shown in figure 18: (solid) shock-curvature term for $\left(y_{20}-R_{s}\right) / r_{2}=0,0.5,1.0$, and 1.5 (dark to light); (symbols) force-coefficient term for $\left(y_{20}-R_{s}\right) / r_{2}=0.5\left(--\triangle^{--}\right)$and $1.5\left(-\cdot--_{-} \cdot-\right)$.

single profile corresponding to $\left(y_{20}-R_{s}\right) / r_{2}=1.5$ diverges from the others, due to the high drag experienced by the secondary as it penetrates deeply into the shock in this case. Overall, however, this approximation may be considered reasonable.

Therefore, (4.3) can now be treated as an autonomous system, and, moreover, the right hand side of (4.3c) is a function only of $\beta$; thus, $\beta$ can be solved for independently of $\eta$ and $v_{\eta}$. We can then construct local phase planes of $\eta$ and $v_{\eta}$ for given values of $\beta$ that can be used to elucidate the behaviour of the secondary trajectories in phase space. It is to be understood, however, that this description is only valid for the single considered value of the initial axial position, $\beta(0)$ (as well as of the initial axial velocity, $\left.\hat{v}_{x 2}(0)-\hat{v}_{x 1}(0)\right)$. Before proceeding with this phase-space analysis, we comment briefly on the differences between (3.3) and (4.3).

The key difference is the introduction in (4.3b) of the shock-curvature term $\left(r_{2} / r_{1}\right)\left[\left(\hat{v}_{x 2}-\right.\right.$ $\left.\hat{v}_{x 1} / \cos \beta\right]^{2} d \beta / d \hat{x}$. Physically, the influence of the shock curvature is to increase the effective magnitude of $v_{\eta}$ as the secondary moves away from the shock, but decrease it as the secondary approaches the shock; thus, the presence of this term will enlarge the region of stability in the local phase-plane. This may also be understood mathematically to arise from the fact that $d \beta / d \hat{x}$ is always negative, so the shock-curvature term will act as a positive contribution to the lift coefficient, decreasing the minimum value of $C_{L 2}$ for which a stable stationary point can exist. This effect is further augmented by the introduction of the primary drag coefficient, $C_{D 1}$, in the force-coefficient term. However, if the combined effect is sufficiently large that $\left(r_{2} / r_{1}\right)\left[\left(\hat{v}_{x 2}-\hat{v}_{x 1}\right) / \cos \beta\right]^{2}|d \beta / d \hat{x}|>\tan \beta\left(1-r_{2} / r_{1}\right) C_{D, f s}$, where $C_{D, f s}$ is the free-stream drag coefficient, then no stable stationary point will exist (this is equivalent to lifting the $L / D$ profile in the left plot of figure 5 such that the free-stream value lies above the horizontal $\tan \beta$ line). In this case, no stable surfing is possible; such a situation will be shown to be realisable, at least locally.

\subsubsection{Phase-space behaviour}

We now return to our phase-space analysis of (4.3). In figure 20 are plotted the phasespace counterparts of the physical trajectories in figure 18 for two radius ratios, $r_{2} / r_{1}=$ 0.05 and 0.2 . In addition, local phase planes of $\eta$ and $v_{\eta}$ are shown for values of $\beta$ corresponding to $\hat{x}=0,1,4$ and 20 . These are evaluated by setting the shock-curvature term in $(4.3 b)$ to its mean value over the four trajectories at the appropriate point 
downstream in each case. The general features of these phase planes are, in part, similar to the sphere-wedge phase diagram in figure 8, with the two stationary points present in all cases. For both radius ratios, however, the stable region in the phase plane is seen to expand considerably in the downstream direction, and this is mirrored in the growing amplitudes of the trajectory oscillations (perhaps more clearly seen in figure 18); this may be attributed to the factors described in the previous paragraph. The growth of the stable region also means that the $r_{2} / r_{1}=0.2,\left(y_{20}-R_{s}\right) / r_{2}=1.5$ trajectory, though beginning slightly outside the local stable region, nevertheless remains stable. Despite this growth, the positions of the stationary points vary but very little downstream: for $r_{2} / r_{1}=0.05$, the stable point, for example, shifts from $\eta=0.76$ at $\hat{x}=0$ to 0.95 at $\hat{x}=20$; for $r_{2} / r_{1}=0.2$, the variation is from 0.73 to 0.96 . The most notable differences between the phase-space pictures for the two radius ratios are the smaller region of stability (most apparent upstream) in the phase plane, and the reduced number of trajectory oscillations, for the larger $r_{2} / r_{1}$. The latter point is obvious from dimensional considerations; the former is discussed shortly.

A significant difference between the present phase diagrams and those of sphere-wedge configurations is the presence of a second centre at $\eta<0$ (for clarity, we now refer to the first centre and its bounding stable region as "principal"). This centre is visible in the $r_{2} / r_{1}=0.2$ planes, but is also present in all those for $r_{2} / r_{1}=0.05$ (though outside the plotted limits) except for that corresponding to $\hat{x}=0$. The appearance of this second stable point is due to the rapid drop in the value of $C_{D 2}$ as $\eta$ decreases inside the shock (see figure 14), which means that the positive $C_{D 1}$ and shock-curvature terms in (4.3b) become dominant, and $d v_{\eta} / d \hat{t}$ switches sign from negative to positive. When this happens, the formerly open phase paths become closed circuits bounding the two stable regions, and it is possible for the secondary to exhibit oscillations that take it from fully inside to fully outside the shock and back again. These predictions should be treated with some caution, however: figure 14 shows that the theoretical model predicts $C_{D 2}$ to drop more rapidly inside the shock than is physically realistic; also, the assumed value of the shock-curvature term in $(4.3 b)$ used to produce these phase planes is likely to be too high for trajectories that penetrate fully into the shocked region.

Phase-space pictures corresponding to those in figure 20 for $M=10$ were also obtained. The main features of the local phase planes are generally similar, but the the primary stable regions are initially slightly smaller for the lower Mach number, and, after reaching a maximum extent near $\hat{x}=4$, begin shrinking in the downstream direction; the oscillations in the secondary trajectories show corresponding reductions in amplitude downstream. One might suspect that these differences are related to the Mach number effect observed in the right plot of figure 5 , as it is reasonable to assume that the extent of the principal stable region in the local phase plane will be determined by the degree to which the maximum $L / D$ value exceeds $\tan \beta$. To investigate whether a similar Mach number effect exists for the sphere-sphere case, on the left axes of figure $21,(L / D)_{\max }$ is plotted against $\tan \beta$ for Mach numbers of 10 and 25 and for various radius ratios. Here $L / D=\left[C_{L 2}-\left(r_{2} / r_{1}\right) C_{L 1}\right] /\left[C_{D 2}-\left(r_{2} / r_{1}\right) C_{D 1}\right]$ and thus is strictly only the effective lift/drag ratio when $\hat{v}_{x 2}-\hat{v}_{x 1}=0$. However, the shock-curvature term varies but very little between these two Mach numbers, so plotting in this form better highlights the differences between the two. The line $(L / D)_{\max }=\tan \beta$ is included for reference, but the location where each curve intersects this line now has little physical significance. As in the sphere-wedge case, $(L / D)_{\max }$ initially lies well above $\tan \beta$ for both Mach numbers and for all radius ratios. As $\beta$ is decreased, however, the $M=10$ profiles drop more steeply, whereas the $M=25$ curves remain well above the $\tan \beta$ line over a large range of $\beta$ (with the ratio $(L / D)_{\max } / \tan \beta$ increasing until $\tan \beta \approx 0.15$ ). This provides a holistic 


$$
18
$$



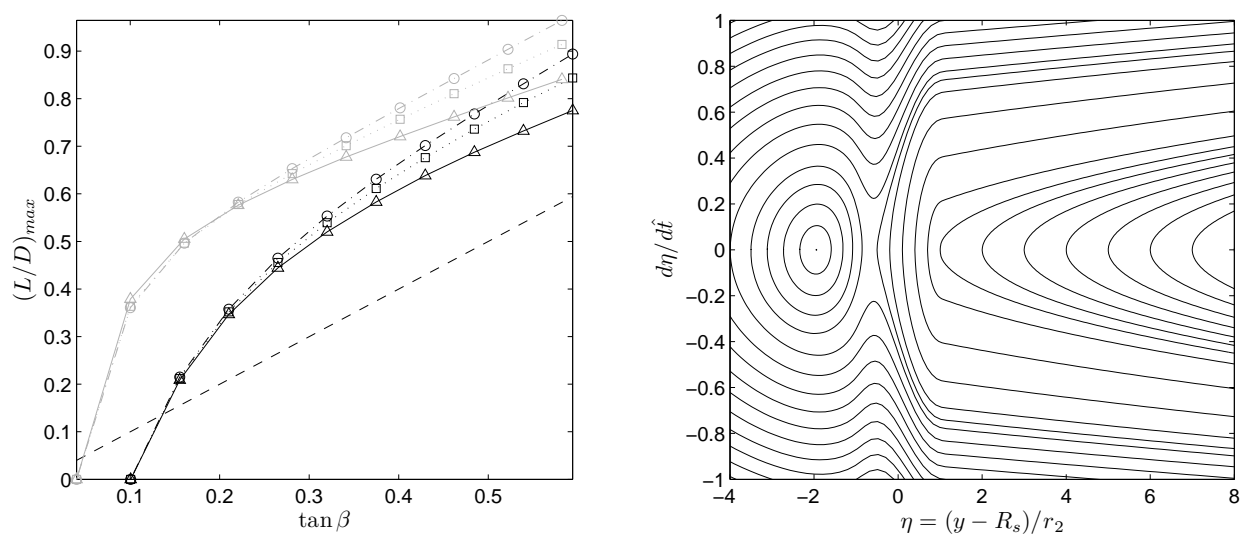

Figure 21. (Left) Maximum lift-to-drag ratios for the secondary sphere at $M=10$ (dark) and $M=25$ (light): $-\cdot--_{-} \cdot-, \quad r_{2} / r_{1}=0.1 ;-\triangle-, \quad r_{2} / r_{1}=0.2 ; \cdots \square \cdots, r_{2} / r_{1}=0.5 ; \quad---$, $(L / D)_{\max }=\tan \beta$. (Right) Phase plane for $M=25, r_{2} / r_{1}=0.2, \hat{x}=4$, using the shock-curvature term for the $\left(y_{20}-R_{s}\right) / r_{2}=1.5$ trajectory in $(4.3 b)$.

explanation as to why the size of the principal stable region decreases in the downstream direction in the $M=10$ phase diagrams but increases for $M=25$. If the Mach number is decreased further, the size of the stable region should continue to shrink; unfortunately, the use of the blast-wave analogy means that the theoretical model cannot be expected to give reliable results for Mach numbers much below 10 .

A further observation from the left plot of figure 21 is that, for a given Mach number and $\beta,(L / D)_{\max }$ is minimum for $r_{2} / r_{1} \approx 0.2$. This explains the decreased extent of the principal stable region for this radius ratio compared to $r_{2} / r_{1}=0.05$ in figure 20 . The reason for this effect is that, as $r_{2} / r_{1}$ is increased, though the peak $C_{L 2}$ decreases monotonically, the influence of the $r_{2} / r_{1} \times C_{D 1}$ term in the denominator of $L / D$ becomes increasingly dominant.

The possibility was mentioned earlier that, if the shock curvature term in $(4.3 b)$ were sufficiently large, the principal stable point could cease to exist. In figure 20, in which the mean value of the shock-curvature term over the relevant trajectories is employed, this phenomenon is not observed. However, it was noted in the discussion of figure 19 that the magnitude of the shock-curvature term for $\left(y_{20}-R_{s}\right) / r_{2}=1.5$ is typically larger than for the other trajectories. On the right axes of figure 21 is shown the phase plane for $M=25, r_{2} / r_{1}=0.2, \hat{x}=4$, using the shock-curvature term corresponding to this single trajectory. The principal stable region is seen to disappear altogether, though the saddle point and the inner stable region remain. A similar picture is obtained for $M=10$. This behaviour helps to explain why the relevant trajectory in figures 18 and 20 shows such large amplitude oscillations. The rapid decay of the shock-curvature term downstream, however, means that the phase planes for both Mach numbers revert to their more familiar appearance by $\hat{x}=20$, and even this trajectory will eventually take the secondary back towards the primary shock.

Finally, we are now in a position to predict the phase-plane picture for initial conditions other than $\hat{x}(0)=0$ and $\hat{v}_{x 2}(0)-\hat{v}_{x 1}(0)=0$. For $\hat{x}(0)<0$ or $\hat{v}_{x 2}(0)-\hat{v}_{x 1}(0) \neq 0$, the magnitude of the shock-curvature term will increase for a given $\beta$, enlargening the principal stable region or transforming the phase plane into the form seen in figure 21. Conversely, for $\hat{x}(0)>0$, the shock-curvature term will become smaller, shrinking the principal region of stability and possibly eliminating the second centre. Even as this term tends to zero, 
however, the principal stable region will remain finitely large except for $\beta$ only slightly greater than the Mach angle. This may be deduced from the left plot of figure 21, in which the intersections of the $(L / D)_{\max }$ curves with the $\tan \beta$ line give the minimum $\beta$ for the existence of a stable region in this limit.

\subsection{Separation of spherical fragments}

As a further application of the theoretical model, we now return to arrangements in which the bodies are initially touching to examine the effects of varying the initial point of alignment and the relative velocities of the bodies. With regard to the meteoroid fragmentation problem, this will give some indication of the influence that the disruption location on the parent body has on the subsequent separation behaviour, and the effects of combining aerodynamic interactions with other mechanisms that can influence the initial separation, such as parent-body rotation.

On the left axes of figure 22, the final (or maximum, for entrainment) normalised separation velocity is plotted against the radius ratio for different choices of the axial alignment point of the spheres: alignment of the centres (as before), of the frontmost point, and of the rearmost point are considered (see figure $2 \mathrm{~d}$; the three of course coincide for $\left.r_{2}=r_{1}\right)$. The initial relative velocity is zero in all cases. First, by comparing the centre-aligned profile with the theoretical $M=25$ profile in figure 17 , the influence of neglecting the repulsive effect of the secondary on the primary (i.e., assigning $C_{L 1}=0$ ) can be deduced. The critical radius ratio is shifted to a slightly higher value, 0.51 compared to 0.48 , and, if expelled, the secondary separation velocity is augmented by a small amount. Comparing the profiles for the three alignment points, the critical radius ratio is observed to increase from 0.19 for front alignment to 0.51 for centre alignment, through to 0.67 for rear alignment. This can be explained by the increase in the effective shock radius experienced by the secondary as it is moved from the front to the rear. The maximum separation velocity also increases as the critical radius ratio decreases, since smaller bodies have smaller ballistic coefficients and are thus more greatly affected by aerodynamic forces. Note that the scaling of Passey \& Melosh, (1.1), gives a result closest to the front-aligned profile, since the assumption of a purely lateral separation is most appropriate here.

We now consider the separation behaviour when there is a finite initial relative velocity between the fragments, $V_{T 0}^{\prime}$. This initial velocity may result, for example, from rotation of the parent body (Passey \& Melosh 1980) or from conversion of the strain energy in the parent body prior to disruption into kinetic energy of the fragments. A rough upper bound for the velocity produced by the latter mechanism can be estimated as follows. The potential strain energy in a body undergoing uniaxial compression is approximately $\varepsilon_{s}=\bar{p}^{2} A l /(2 E)=\bar{p}^{2} m_{0} /\left(2 \rho_{m} E\right)$, where $A, l$ and $m_{0}$ are the frontal area, length, and mass of the body, $\bar{p}$ is the mean pressure, and $E$ is the Youngs modulus. If this energy is assumed to be converted entirely into lateral kinetic energy of the fragment pair, conservation of momentum gives $\left(1+m_{2} / m_{1}\right) m_{2} V_{T}^{2}=2 \varepsilon_{s}$. Using $m_{0} / m_{2}=1+m_{1} / m_{2}$, we then obtain

$$
\frac{V_{T 0, \max }}{V}=\left(\frac{1}{\rho_{m} E} \frac{1+\left(r_{1} / r_{2}\right)^{3}}{1+\left(r_{2} / r_{1}\right)^{3}}\right)^{1 / 2} \rho_{a} V .
$$

The maximum separation velocity from this mechanism thus increases approximately as $\left(r_{2} / r_{1}\right)^{-3 / 2}$ as $r_{2} / r_{1}$ is decreased.

If both the magnitude and the direction of the initial separation velocity are allowed to vary, as well as the initial alignment of the fragments, the parameter space becomes too large to investigate. We thus limit ourselves to purely lateral initial separations 

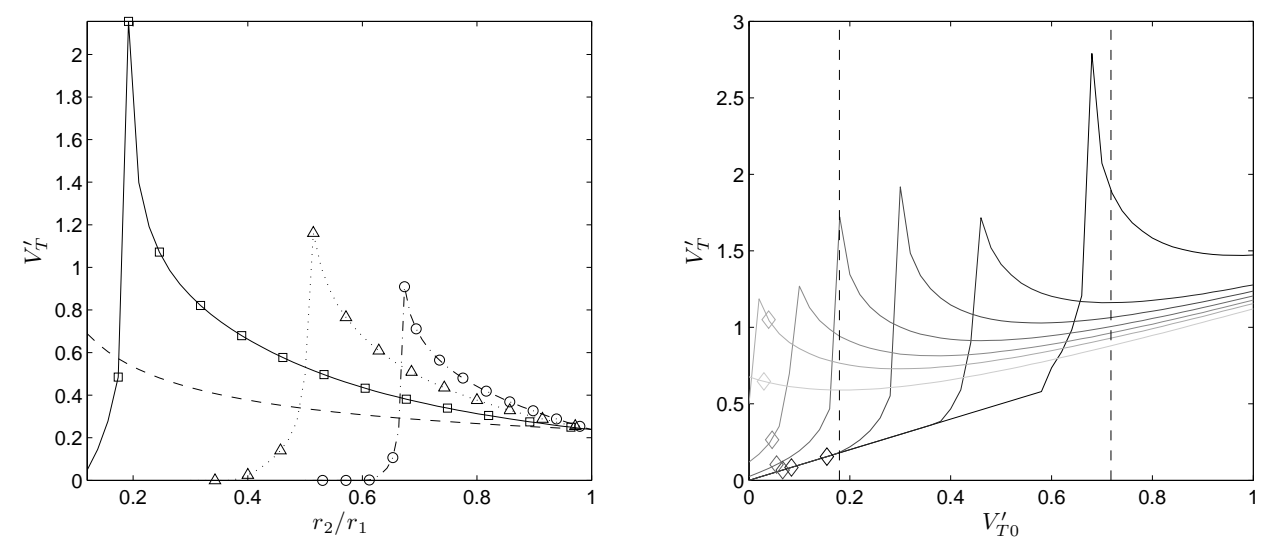

FIGURE 22. Normalised separation velocities calculated for initially touching spheres at a Mach number of $M=25$, using the theoretical model. (Left) Zero initial separation velocity with: $-\cdot--_{-} \cdot-$, rearmost points aligned; $\cdots \triangle \cdots$, centres aligned; $-\square-$, frontmost points aligned; --- , scaling indicated by (1.1). (Right) Nonzero initial lateral velocities with sphere centres axially aligned, for (darkest to lightest): $r_{2} / r_{1}=0.2,0.3,0.35,0.4,0.45,0.5$, and $0.6 ; \diamond$, maximum separation velocity from strain-energy conversion for the Sikhote-Alin meteoroid, according to (4.4). The vertical dashed lines indicate the maximum initial velocity from a spinning parent body, with parameters appropriate for the Sikhote-Alin meteoroid, assuming rotation rates of 1 and 4 rps.

and axial alignment of the sphere centres (figure 2e). On the right axes of figure 22 is plotted the final (or maximum, for entrainment) normalised separation velocity versus the initial normalised velocity, $V_{T 0}^{\prime}=\sqrt{\rho_{m} / \rho_{a}} V_{T 0} / V$, for a number of choices of $r_{2} / r_{1}$. As $V_{T 0}^{\prime}$ is increased, extended surfing becomes possible for smaller radius ratios, leading to a decreased critical $r_{2} / r_{1}$. This is similar to the effect of moving the alignment point forward, as discussed earlier; the corresponding increase in the maximum separation velocity is also generally observed (note, however, that the precise value of the maximum for a given $r_{2} / r_{1}$ is sensitive to the choice of initial conditions, so the peak in each profile should be considered a lower bound). On each of the curves is also indicated the point corresponding to the maximum initial separation velocity that is available from strain-energy conversion for the relevant radius ratio, (4.4), for parameters appropriate for the Sikhote-Alin meteoroid at breakup (Krinov 1966). Since this meteoroid underwent multiple stages of fragmentation, the height of the second-to-last stage according to eyewitness accounts $(16 \mathrm{~km})$, which was most likely responsible for the major dimensions of the crater field, is chosen, giving an atmospheric density of $\rho_{a}=0.17 \mathrm{~kg} / \mathrm{m}^{3}$. The values of the other parameters are $V=15 \mathrm{~km} / \mathrm{s}$, and $E=200 \mathrm{GPa}$. Strain-energy conversion is seen to have a small effect on the separation behaviour: even for this limiting case, the critical radius ratio drops by only a small amount to slightly below 0.5 . For weaker bodies (e.g., stones), the reduced $E$ in (4.4) will be offset by a decreased $\rho_{a}$ at the point of disruption, and these results will not be substantially altered.

Also shown on the right plot of figure 22 are vertical lines corresponding to the maximum initial separation velocities resulting from rotation rates of $\omega_{0}=1$ and $4 \mathrm{rps}$ for the Sikhote-Alin parent body. These assume an initial mass of 300 tonnes (Heide 1963), and thus, with a density of $\rho_{m}=7800 \mathrm{~kg} / \mathrm{m}^{3}$, a radius of approximately $2 \mathrm{~m}$. The effect of spinning on the subsequent surfing behaviour is seen to be potentially much more significant for meteoroids of this size than strain-energy conversion: for 1 and $4 \mathrm{rps}$, the critical radius ratio drops to 0.4 and $\sim 0.2$, respectively. Moving the initial point of alignment 


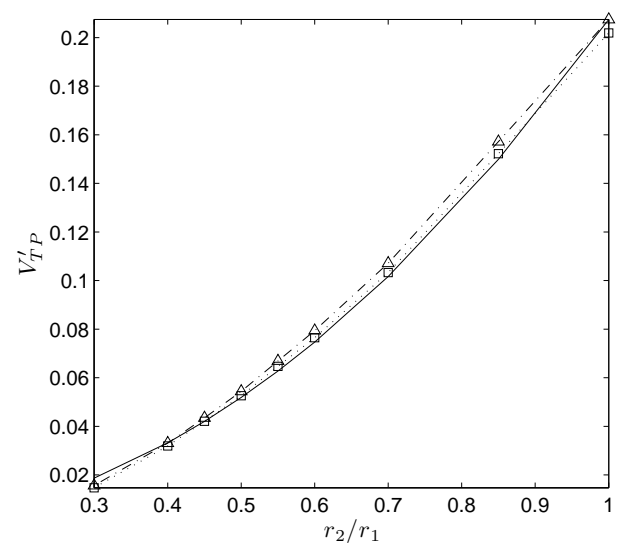

Figure 23. Normalised separation velocity of the primary body from numerical simulations of initially touching two-sphere configurations: $-\cdot-\triangle^{-\cdot-}, M=10 ; \cdots \square \cdots, M=25$; $V_{T P}^{\prime} \propto\left(r_{2} / r_{1}\right)^{2}$

forward, or increasing the parent body radius, would decrease the critical ratio even further. These results also show that the combined effects of surfing and a modest rotation rate give the potential for a significant variation in the final separation velocity. The maximum separation velocity for $r_{2} / r_{1}=0.2$ in the right plot of figure 22 corresponds to a value of $C=1.56$ in (1.1); thus, a large fraction of the variation deduced in this "constant" by Passey \& Melosh from terrestrial crater fields (see $\S 1$ ) can be explained by the combination of these two mechanisms.

\subsection{Primary body motion for initially touching configurations}

In previous separate-fragment models (Artemieva \& Shuvalov 2001; Bland \& Artemieva 2006), the separation behaviour of the primary body was derived from conservation of lateral momentum applied to the fragment pair. This assumption, while valid for equally sized fragments (but from symmetry rather than mechanical considerations), was proposed by the authors with some doubts as to its validity when $r_{2} \neq r_{1}$. Indeed, both the presence of intervening fluid (to which momentum may be transferred) and the limited domain of influence of a body in supersonic flow may lead one to question this assumption, even without knowledge of the surfing phenomenon. With the highly asymmetrical domains of influence produced by surfing, however, it is clearly not appropriate to correlate the final lateral momenta of the two bodies. In the computations described in $\S 4.3$, for example, the magnitude of the maximum lateral momentum of the secondary varies from less than a third of to more than twice that of the primary.

The theoretical model developed in this work does not tell us anything about the motion of the primary during separation from an initially touching configuration, and to obtain quantitative results we must look to the relevant computations. These can still be informed by general physical arguments, however. Following a similar line of reasoning to Passey \& Melosh (1980), we write

$$
V_{T P}=\bar{F}_{T P} \Delta t / m_{1},
$$

where $V_{T P}$ is the final separation velocity of the primary, $\bar{F}_{T P}$ is the mean lateral force, and $\Delta t$ is the time period over which the secondary influences the primary. Assuming the separation to be dominated by the secondary motion, we may characterise the influence time as $\Delta t=\sqrt{2 \xi / \alpha_{2}}$, where $\xi$ and $\alpha_{2}$ are the secondary influence distance and separation 
acceleration, respectively. The influence distance will scale as $\xi \propto r_{2}^{k}$, where $0 \lesssim k \lesssim 1$ : the upper limit for $k$ is appropriate for $r_{2} \approx r_{1}$, in which case the separation is primarily lateral and the influence distance scales with the secondary shock radius; for $r_{2} \ll r_{1}$, however, the separation is principally axial due to the significantly lower ballistic coefficient of the secondary, and the dependence of $\xi$ on $r_{2}$ will be weak. In either case, the secondary acceleration will scale roughly as $\alpha_{2} \propto 1 / r_{2}$. The lateral force exerted by the secondary on the primary will also depend on the secondary size, as a larger secondary will have a larger pressure footprint on the primary surface. If the primary surface were planar, this would result in $\bar{F}_{T P} \propto r_{2}^{2}$ (which will be the limiting behaviour as $r_{2} / r_{1} \rightarrow 0$ ), but, because of the the curvature of the primary surface, the dependence will, in general, be less than quadratic.

Combining these dependencies, we have $V_{T P} \propto r_{2}^{m}$, where $1 \leqslant m \leqslant 3$, and with $m \approx 2$ likely to be an appropriate value over a range of $r_{2} / r_{1}$. Indeed, in figure 23 , the normalised primary separation velocities from the free-flying simulations described in $\S 4.3$ are plotted, and a value of $m=2$ in the scaling of $V_{T P}$ is seen to provide a good fit to the data. Although these arguments are not sufficient in themselves to provide quantitative information regarding the primary separation behaviour, with a small number of relevant computations, they can be used to extrapolate the primary behaviour over a range of relative body sizes.

\section{Discussion}

We conclude with a brief discussion of the implications of the shock-surfing phenomenon, particularly for meteoroid fragmentation. First, because of the dependence of the extent of surfing on the radius ratio in initially touching configurations, and the highly augmented separation velocities that can result from extended surfing, it is clear that the radius ratio is a critical parameter in determining the separation behaviour. The influence of the radius ratio is not well captured by the scaling law of Passey \& Melosh, (1.1), and, moreover, the extrapolation of the separation behaviour of equally sized fragments to unequal fragments, as in previous separate-fragment models (Artemieva \& Shuvalov 2001; Bland \& Artemieva 2006), is unlikely to yield accurate results. The implications of surfing for the assumption of conserved lateral momentum in deriving the primary motion in these models has also been discussed.

The existence of a critical radius ratio (or, more correctly, a critical ratio range, given that a number of factors affect the precise value of this ratio), delineating entrainment of the secondary within the primary shock from expulsion, will also lead to a selection effect for multiple fragments travelling together, e.g., immediately following atmospheric disruption of the parent body. The largest fragments will repulse one another, but with moderate resulting separation velocities; slightly smaller fragments will have the potential to be repulsed strongly due to surfing effects; whereas the smallest fragments will likely be entrained within the shocks of larger fragments (at which point a further selection effect can take place due to the dependence of the lateral force coefficient on the radius ratio noted by Laurence et al. (2007)). This could lead to interesting and observable phenomena in the formation of crater fields: for example, outlying fragments could be produced by extended surfing immediately following disruption, and smaller fragments could impact further downrange than would otherwise be possible due to their travelling within the low-density region behind larger fragments. Regarding this last point, one of the "peculiarities" of the Sikhote-Alin crater field noted by Krinov (1966) is that "a fairly large number of small craters and holes were found to lie in the front part between 
large craters." This may in fact not be a peculiarity at all, but an expected result of interactions between fragments of different sizes.

Finally, the impinging shock interactions experienced during surfing will lead to extremely high local pressures and heat fluxes on the secondary body (Edney 1968; Sanderson et al. 2004). Such interactions, for example, were responsible for burning "gaping holes" in the pylon of a dummy ramjet attached to the X-15 aircraft (Bertin \& Cummings 2006). This increased dynamic and thermal loading would lead to enhanced ablation and possibly further fragmentation of the impinged-upon fragment, and would also increase the rate of energy transfer to the atmosphere.

S.J.L. was a postdoctoral scholar at the Graduate Aeronautical Laboratories, California Institute of Technology, under the supervision of Professor Hans Hornung, when this work began, and is grateful for many fruitful discussions with Professor Hornung on the subject. S.J.L. also wishes to acknowledge H.J. Melosh, whose critique of an earlier work helped lead to the present manuscript. The computations in Section 3 were carried out by R.D. at the Oak Ridge National Laboratory, which is managed by UT-Battelle, LLC under Contract No. DE-AC05-00OR22725.

\section{REFERENCES}

Artem'eva, N. A. \& Shuvalov, V. V. 1996 Interaction of shock waves during the passage of a disrupted meteoroid through the atmosphere. Shock Waves 5, 359-367.

Artemieva, N. A. \& Pierazzo, E. 2009 The Canyon Diablo impact event: projectile motion through the atmosphere. Meteorit. Planet. Sci. 44 (1), 25-42.

Artemieva, N. A. \& Shuvalov, V. V. 2001 Motion of a fragmented meteoroid through the planetary atmosphere. J. Geophys. Res. 106 (E2), 3297-3309.

BARRI, N. G. 2009 Aerodynamic interaction of meteor-body fragments: the collimation effect. Dokl. Phys. 54 (9), 423-425.

Berger, M. \& Colella, P. 1988 Local adaptive mesh refinement for shock hydrodynamics. J. Comput. Phys. 82, 64-84.

Bertin, J. J. \& Cummings, R. M. 2006 Critical hypersonic aerothermodynamic criteria. Ann. Rev. Fluid Mech. 38, 129-157.

BiLlig, F. S. 1967 Shock-wave shapes around spherical- and cylindrical-nosed bodies. J. Spacecraft Rockets 4 (6), 822-823.

Bland, P. A. \& ARtemieva, N. A. 2003 Efficient disruption of small asteroids by Earth's atmosphere. Nature 424, 288-291.

Bland, P. A. \& Artemieva, N. A. 2006 The rate of small impacts on Earth. Meteorit. Planet. Sci. 41 (4), 607-631.

Chyba, C. F., Thomas, P. J. \& Zahnle, K. J. 1993 The 1908 Tunguska explosion: atmospheric disruption of a stony asteroid. Nature 361, 40-44.

Cirak, F., Deiterding, R. \& Mauch, S. P. 2007 Large-scale fluid-structure interaction simulation of viscoplastic and fracturing thin shells subjected to shocks and detonations. Computers 85 Structures 85 (11-14), 1049-1065.

DeITERDing, R. 2003 Construction and application of an AMR algorithm for distributed memory computers. In Adaptive Mesh Refinement - Theory and Applications, Lecture Notes in Computational Science and Engineering 41, pp. 361-372.

Deiterding, R. 2005 Detonation structure simulation with AMROC. In High Performance Computing and Communications 2005, Lecture Notes in Computer Science 3726, pp. 916927.

Deiterding, R. 2009 A parallel adaptive method for simulating shock-induced combustion with detailed chemical kinetics in complex domains. Computers 83 Structures 87, 769-783.

Deiterding, R., Cirak, F. \& Mauch, S.P. 2009 Efficient fluid-structure interaction simulation of viscoplastic and fracturing thin-shells subjected to underwater shock loading. In S. Hartmann et al., eds., Int. Workshop on Fluid-Structure Interaction, pp. 65-80. University Press GmbH, Kassel. 
Deiterding, R., Radovitzky, R., Mauch, S. P., Noels, L., Cummings, J. C. \& Meiron, D. I. 2005 A Virtual Test Facility for the efficient simulation of solid materials under high energy shock-wave loading. Engineering Comput. 22 (3-4), 325-347.

EDNEy, B. E. 1968 Effects of shock impingement on the heat transfer around blunt bodies. AIAA J. 6 (1), 15-21.

Fedkiw, R. P., Aslam, T., Merriman, B. \& Osher, S. 1999 A non-oscillatory Eulerian approach to interfaces in multimaterial flows (the ghost fluid method). J. Comput. Phys. 152, 457-492.

Hayes, D. H. \& Probstein, R. R 1966 Hypersonic Flow Theory. New York: Academic Press.

HeIde, F. 1963 Meteorites. Chicago: University of Chicago Press.

Hills, J. G. \& GODA, M. P 1993 The fragmentation of small asteroids in the atmosphere. Astron. J. 105 (3), 1114-1144.

Krinov, E. L. 1966 Giant Meteorites, translated by J.S. Romankiewicz. New York: Pergamon Press.

Laurence, S. J. 2006 Proximal bodies in hypersonic flow. PhD thesis, California Institute of Technology, Pasadena, California.

Laurence, S. J., Deiterding, R. \& Hornung, H. G. 2007 Proximal bodies in hypersonic flow. J. Fluid Mech. 590, 209-237.

LeEs, L. 1955 Hypersonic flow. In Proc. 5th Int. Conf. Los Angeles, pp. 241-276. Inst. Aero. Soc., New York.

PAssey, Q. R. \& Melosh, H. J. 1980 Effects of atmospheric breakup on crater field formation. Icarus 42, 211-233.

Sanderson, S. R., Hornung, H. G. \& Sturtevant, B. 2004 The influence of non-equilibrium dissociation on the flow produced by shock impingement on a blunt body. J. Fluid Mech. 516, $1-37$.

Schultz, P. H. \& Sugita, S. 1994 Penetrating and escaping the atmospheres of Venus and Earth. In Lunar and Planetary Science Conference XXV, pp. 1215-1216.

Sedov, L. I. 1959 Similarity and dimensional methods in mechanics. Translation edited by Maurice Holt. Translation by Morris Friedman from the 4th Russian ed. New York: Academic Press.

Svetsov, V. V., Nemtchinov, I. V. \& Teterev, A. V. 1995 Disintegration of large meteoroids in Earth's atmosphere: theoretical models. Icarus 116, 131-153.

Toro, E. F. 1999 Riemann solvers and numerical methods for fluid dynamics. Berlin, Heidelberg: Springer-Verlag.

Vincenti, W. G. \& Kruger, C. H. 1965 Introduction to physical gas dynamics. Florida: Krieger. 